ALEA, Lat. Am. J. Probab. Math. Stat. 15, 1027-1063 (2018)

DOI: $10.30757 /$ ALEA.v15-38

\title{
Conditional decoupling of random interlacements
}

\author{
Caio Alves and Serguei Popov \\ Mathematics Institute, University of Leipzig, Augustusplatz 10, Leipzig, Germany \\ E-mail address: caio.alves@math.uni-leipzig.de \\ Department of Statistics, Institute of Mathematics, Statistics and Scientific Computation, \\ University of Campinas - UNICAMP, Rua Sérgio Buarque de Holanda 651, 13083-859, \\ Campinas SP, Brazil \\ E-mail address: popov@ime.unicamp.br \\ URL: http://www.ime.unicamp.br/ p popov/
}

\begin{abstract}
We prove a conditional decoupling inequality for the model of random interlacements in dimension $d \geq 3$ : the conditional law of random interlacements on a box (or a ball) $A_{1}$ given the (not very "bad") configuration on a "distant" set $A_{2}$ does not differ a lot from the unconditional law. The main method we use is a suitable modification of the soft local time method of Popov and Teixeira (2015), that allows dealing with conditional probabilities.
\end{abstract}

\section{Introduction and results}

Random interlacements were introduced by Sznitman (2010), to model the trace of the simple random walk on the discrete torus $\mathbb{Z}_{n}^{d}:=\mathbb{Z}^{d} / n \mathbb{Z}^{d}$ or the discrete cylinder $\mathbb{Z} \times \mathbb{Z}^{d-1}$, in dimension $d \geq 3$. Detailed treatments and reviews of recent results can be found in the recent books Cerný and Teixeira (2012); Drewitz et al. (2014); Sznitman (2012c). Loosely speaking, the model of random interlacements in $\mathbb{Z}^{d}, d \geq 3$, is a stationary Poissonian soup of bi-infinite simple random walk trajectories on the integer lattice. There is a parameter $u>0$ entering the intensity measure of the Poisson process, the larger $u$ is the more trajectories are thrown in. The sites of $\mathbb{Z}^{d}$ that are not touched by the trajectories constitute the vacant set $\mathcal{V}^{u}$, and the union of all trajectories constitutes the interlacement set $\mathcal{I}^{u}=\mathbb{Z}^{d} \backslash \mathcal{V}^{u}$. The random interlacements are constructed simultaneously for all $u>0$ in such a way that $\mathcal{I}^{u_{1}} \subset \mathcal{I}^{u_{2}}$ if $u_{1}<u_{2}$. In fact, the law of the vacant set at level $u$ can be uniquely characterized by the following identity:

$$
\mathbb{P}\left[A \subset \mathcal{V}^{u}\right]=\exp (-u \operatorname{cap}(A)),
$$

Received by the editors September 7th, 2017; accepted May 20th, 2018.

2010 Mathematics Subject Classification. Primary 60K35; secondary 60G50, 82C41.

Key words and phrases. Random interlacements, stochastic domination, soft local time. 
where $\operatorname{cap}(A)$ is the capacity of a finite set $A \subset \mathbb{Z}^{d}$. Informally, the capacity measures how "big" is the set from the point of view of the walk, see Section 6.5 of Lawler and Limic (2010) for formal definitions, or Section 2 below.

The model of random interlacements naturally has more independence built in than just one random walk on the torus or the cylinder (because on a fixed set one observes traces of independent trajectories). Still, the analysis of random interlacements is difficult because of the long-range dependencies present there. For example, in (1.68) from Sznitman (2010) we can see that

$$
\operatorname{Cov}\left(\mathbb{1}_{x \in \mathcal{I}^{u}}, \mathbb{1}_{y \in \mathcal{I}^{u}}\right) \sim \frac{c_{d} u}{\|x-y\|^{d-2}} \quad \text { as } \quad\|x-y\| \rightarrow \infty,
$$

which means that the "degree of dependence" decreases polynomially in the distance.

Naturally, one is interested in "decoupling" the events supported on distant regions; that is, to argue that they are approximately independent to a certain degree. One possible approach to quantify that degree is the following: given finite sets $A_{1}, A_{2} \subset \mathbb{Z}^{d}$ and functions $f_{1}:\{0,1\}^{A_{1}} \rightarrow[0,1]$ and $f_{2}:\{0,1\}^{A_{2}} \rightarrow[0,1]$ depending on the interlacements set intersected with $A_{1}$ and $A_{2}$ respectively, we have

$$
\operatorname{Cov}_{u}\left(f_{1}, f_{2}\right) \leq c_{d} u \frac{\operatorname{cap}\left(A_{1}\right) \operatorname{cap}\left(A_{2}\right)}{\operatorname{dist}\left(A_{1}, A_{2}\right)^{d-2}},
$$

as proved in formula (2.15) of Sznitman (2010), see also (8.1.1) in Drewitz et al. (2014). However, the polynomial error term in (1.3) can complicate one's life in many applications (and, e.g. in the case when the diameters of these sets are of the same order as the distance between them, (1.3) is simply of no use); on the other hand, while (1.3) can be improved to some degree (de Bernardini et al., 2018+), the error term there should always be at least polynomial, as (1.2) shows. To circumvent this difficulty, one first may note that usually the "interesting" events/functions are monotone (i.e., increasing or decreasing). For e.g. increasing events, we know that their probabilities increase as the parameter $u$ increases. Note also that the FKG inequality (see Teixeira, 2009, Theorem 3.1) gives us

$$
\mathbb{E}^{u}\left[g_{1} g_{2}\right] \geq \mathbb{E}^{u}\left[g_{1}\right] \mathbb{E}^{u}\left[g_{2}\right]
$$

for any increasing functions $g_{1}, g_{2}$ with finite second moments. To complement the FKG inequality, we use sprinkling, i.e., we slightly change the intensity of random interlacements in order to decrease the error term; this approach was used in Sznitman (2010) and Sznitman (2012a). Then, in particular, in Popov and Teixeira (2015) it was proved that

$$
\mathbb{E}^{u}\left[f_{1} f_{2}\right] \leq \mathbb{E}^{(1+\varepsilon) u}\left[f_{1}\right] \mathbb{E}^{(1+\varepsilon) u}\left[f_{2}\right]+c_{d}(r+s)^{d} \exp \left(-c_{d}^{\prime} \varepsilon^{2} u s^{d-2}\right) ;
$$

with $f_{1}:\{0,1\}^{A_{1}} \rightarrow[0,1]$ and $f_{2}:\{0,1\}^{A_{2}} \rightarrow[0,1]$ both increasing functions in the interlacements set, $r=\min \left(\operatorname{diam}\left(A_{1}\right), \operatorname{diam}\left(A_{2}\right)\right)$, and $s=\operatorname{dist}\left(A_{1}, A_{2}\right)$. The same bound was also obtained for decreasing functions.

It is important to observe, however, that the decoupling in the above form may not always be useful for one's needs. Intuitively, one is tempted to understand inequalities like (1.3) as "what happens in one set does not influence a lot what happens in the other set". Now, consider the following situation. Suppose that on top of the random interlacements we have some additional stochastic process (e.g., a random walk) that "explores" the interlacement set in some way. Assume 
that this process has already explored the interlacements in a given area, revealing a lot of information about it; think, for definiteness, that it simply revealed the interlacement set exactly. The probability of a particular configuration of the interlacement set is usually very small; so, (1.3) (even (1.5)!) will blow up when one divides by that probability, because of the error term. In fact, in the end of Section 2 we discuss a particular model of the random walk on the interlacement set, where our main results turn out to be useful.

This justifies the need for conditional decoupling, i.e., show that, given the configuration on some set, the law of the interlacement configuration on a distant set is still in some sense close to the unconditional law. This is what we are doing in this paper. To prove our results, the main method we use is a suitable modification (that allows dealing with conditional probabilities) of the soft local time method of Popov and Teixeira (2015). We hope that this modification will be useful in other contexts, for instance, for dealing with the decoupling properties of the loop measures Chang and Sapozhnikov (2016).

Another important observation is the following. There are strong connections between random interlacements and the Gaussian free field, see e.g. Sznitman (2012b,c). In particular, there are decoupling inequalities similar to (1.3) and (1.5) for the Gaussian free field as well, see Popov and Ráth (2015). Notice, however, that the decoupling-with-sprinkling result for the Gaussian free field (Theorem 1.2 of Popov and Ráth, 2015) is already conditional (the unconditional decoupling is obtained as a simple consequence, just by integration). On the other hand, note that the error terms in the conditional decoupling in the main result of this paper (Theorem 1.1) are much worse than that of (1.5); related to this is the fact that in the conditional setting the minimal distance between sets that permits the result to work is much bigger. A comparison with the situation for the Gaussian free field suggests that, hopefully, there is still much room for improvement for the conditional decoupling for random interlacements.

Let us briefly introduce a bit of notation in order to state our main result. Let $r>0$ be sufficiently big, and let $s:=s(r)>0$, with $s=o(r)$. We define $A_{1}^{\circ}:=A_{1}^{\bigcirc}(r)$ to be the discrete Euclidean ball of radius $r$ and center at the origin, that is

$$
A_{1}^{\circ}:=\left\{x \in \mathbb{Z}^{d} ; \operatorname{dist}(x, 0) \leq r\right\},
$$

where $\operatorname{dist}(\cdot, \cdot)$ denotes de Euclidean distance. We also define $A_{1}^{\square}:=A_{1}^{\square}(r)$ as a $d$-dimensional hypercube with side-length $2 r+1$ and center at the origin, that is

$$
A_{1}^{\square}:=\left\{x \in \mathbb{Z}^{d} ;\|x\|_{\infty} \leq r\right\},
$$

where $\|\cdot\|_{\infty}$ denotes the $\ell_{\infty}$ norm. We then define the sets

$$
\begin{aligned}
& A_{2}^{\circ}=A_{2}^{\circ}(r, s):=\left\{x \in \mathbb{Z}^{d} ; \operatorname{dist}(x, 0)>r+2 s\right\}, \\
& A_{2}^{\square}=A_{2}^{\square}(r, s):=\left\{x \in \mathbb{Z}^{d} ;\|x\|_{\infty}>r+2 s\right\} .
\end{aligned}
$$

When there is no risk of confusion, or when the arguments presented work for both balls and smoothed hypercubes (which will be often so), we will omit the super-indexes $\circ, \square$.

We will now state our main result. Heuristically, it says the following: Let $s$ be bounded from below by a power of $r$ with an explicit given coefficient (strictly smaller than 1, depending only on the dimension $d$ and whether $A_{1}$ is a ball or a hypercube). Let $A_{3}$ be a subset of $A_{2}$ with finite boundary, that is, $A_{3}$ is either 


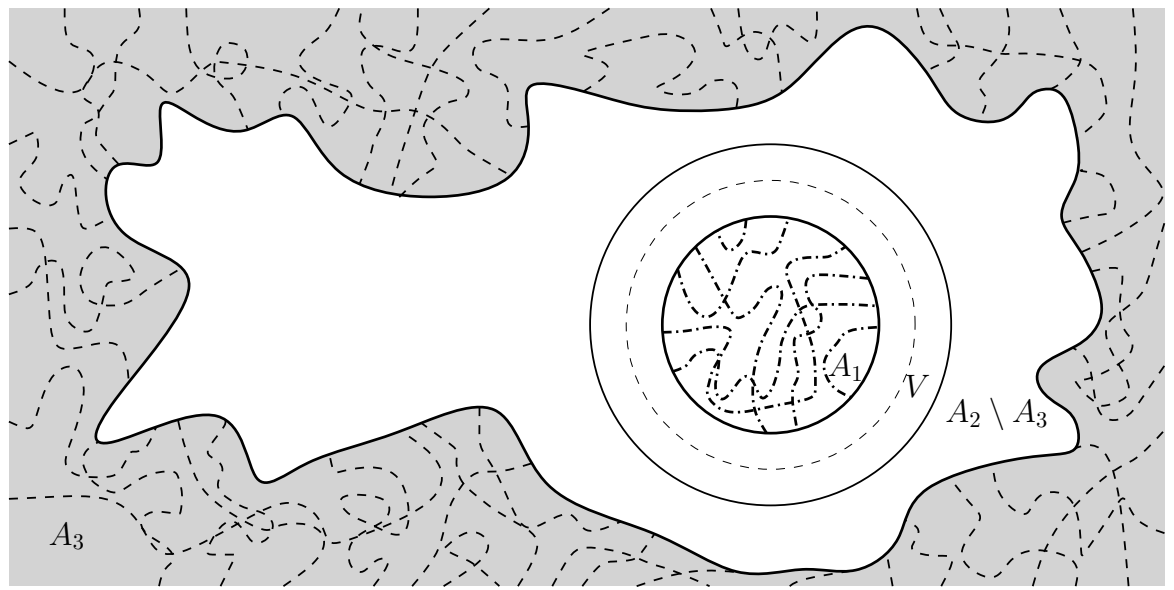

FiguRE 1.1. Our main result says that if the interlacements configuration in a set $A_{3} \subseteq A_{2}$ is not too weird, that is, it does not belong to a set with stretched exponentially small probability (in $s$, as $s \rightarrow \infty$ ), then with high probability (1 minus stretched exponential in $s$ ) the distribution of the interlacements set intersected with $A_{1}$ conditioned on the state of $\mathcal{I}^{u} \cap A_{3}$ can be well approximated by the unconditional distribution.

finite or has finite complement. If we pay a stretched exponentially small price (in $s$ ) to guarantee that the interlacements configuration of $\mathcal{I}^{u} \cap A_{3}$ is not too weird, then the distribution of $\mathcal{I}^{u} \cap A_{1}$ conditioned on this configuration is well approximated by the unconditional distribution, with high probability (1 minus a stretched exponential function of $s$ ).

Theorem 1.1. Let the real numbers $b_{\odot}, b_{\square}$ be such that

$$
\begin{aligned}
& 1 \leq b_{\circ}<\frac{2 d-2}{d}, \\
& 1 \leq b_{\square}<\frac{4 d-4}{3 d-2} .
\end{aligned}
$$

Then, define

$$
\begin{aligned}
& a_{\odot}=2 d-2-d b_{\odot}>0, \\
& a_{\square}=4 d-4-3 d b_{\square}+2 b_{\square}>0 .
\end{aligned}
$$

From now on we will again omit the indexes $\bigcirc, \square$. Recall that $r$ is of the same order as the diameter of $A_{1}$, and that $s$ has the same order as the distance between $A_{1}$ and $A_{2}$. Assume $s=s(r) \asymp r^{\frac{1}{b}}$, let $r$ be sufficiently big. Let $\varepsilon>0$ be smaller than $1 / 4$. Let $A_{3}$ be a subset of $A_{2}$ such that $\left|\partial A_{3}\right|<\infty$. Define $\mathcal{I}_{A_{j}}^{u}:=\mathcal{I}^{u} \cap A_{j}$, for $j=1,2,3$.

Then there are positive constants $c, c^{\prime}$ depending only on the dimension $d$, and a measurable (according to a suitably constructed $\sigma$-field) set $\mathcal{G} \in\{0,1\}^{A_{3}}$ such that

$$
\mathbb{P}^{u}\left[\mathcal{I}_{A_{3}}^{u} \in \mathcal{G}\right] \geq 1-\exp \left(-\frac{c^{\prime}}{2} \varepsilon^{2} u s^{a}\right),
$$


and for any increasing function $f$ on the interlacements set intersected with $A_{1}$, with $\|f\|_{\infty} \leq 1$, we have

$$
\begin{aligned}
\left(\mathbb{E} f\left(\mathcal{I}_{A_{1}}^{u(1-\varepsilon)}\right)-c \exp \left(-c^{\prime} \varepsilon^{2} u s^{a}\right)\right) \mathbb{1}_{\mathcal{I}_{A_{3}}^{u} \in \mathcal{G}} \leq \mathbb{E}\left(f\left(\mathcal{I}_{A_{1}}^{u}\right) \mid \mathcal{I}_{A_{3}}^{u}\right) \mathbb{1}_{\mathcal{I}_{A_{3}}^{u} \in \mathcal{G}} \\
\leq\left(\mathbb{E} f\left(\mathcal{I}_{A_{1}}^{u(1+\varepsilon)}\right)+c \exp \left(-c^{\prime} \varepsilon^{2} u s^{a}\right)\right) \mathbb{1}_{\mathcal{I}_{A_{3}}^{u} \in \mathcal{G}}
\end{aligned}
$$

We also obtain a result analogous to Theorem 1.1, but this time we allow the sprinkling factor to be arbitrarily big. This decreases the "precision" (in the result below, $\mathbb{E} f\left(\mathcal{I}_{A_{1}}^{u+u^{\prime}}\right)$ can be very different from $\mathbb{E} f\left(\mathcal{I}_{A_{1}}^{u}\right)$ ), but, in compensation, the size of the complement of the "good" set as well as the "error term" become smaller.

Theorem 1.2. Let $u^{\prime}>u>0$. We use the same definitions as in Theorem 1.1. There are positive constants $c, c^{\prime}$ depending only on the dimension $d$, and a measurable (according to the random interlacements $\sigma$-field) set $\mathcal{G}_{u^{\prime}} \in\{0,1\}^{A_{3}}$ such that

$$
\mathbb{P}^{u}\left[\mathcal{I}_{A_{3}}^{u} \in \mathcal{G}_{u^{\prime}}\right] \geq 1-\exp \left(-c^{\prime} u^{\prime} s^{a}\right),
$$

and for any increasing function $f$ on the interlacements set intersected with $A_{1}$, with $\|f\|_{\infty} \leq 1$, we have

$$
\mathbb{E}\left(f\left(\mathcal{I}_{A_{1}}^{u}\right) \mid \mathcal{I}_{A_{3}}^{u}\right) \mathbb{1}_{\mathcal{I}_{A_{3}}^{u} \in \mathcal{G}_{u^{\prime}}} \leq\left(\mathbb{E} f\left(\mathcal{I}_{A_{1}}^{u+u^{\prime}}\right)+c \exp \left(-c^{\prime} u^{\prime} s^{a}\right)\right) \mathbb{1}_{\mathcal{I}_{A_{3}}^{u} \in \mathcal{G}_{u^{\prime}}}
$$

Remark 1.3. We have to explain why we need to consider $A_{3} \subset A_{2}$. Indeed, at first sight it seems that conditioning on a configuration on $A_{3}$ does not add generality to our results, since any fixed configuration on $A_{3}$ corresponds to a set of configurations on $A_{2}$. However, the problem with always setting $A_{3}=A_{2}$ is the following: the "exceptional set" $\mathcal{G}^{c}$ will then be supported on the whole $A_{2}$, and this can be inconvenient for applications. For example, assume that we successively apply the conditional decoupling results to a process (such as the one of Section 1.1) that "explores" the interlacement environment. If that process has explored only a finite chunk of $A_{2}$, we would not be able to say if the configuration is "good" (i.e., belongs to $\mathcal{G})$ by only observing that finite chunk. This would force us to condition on the (configuration on the) whole $A_{2}$, which would mean that a subsequent application of a conditional decoupling may be difficult, since we already "revealed" some information about the configuration on a set which is "too big" (i.e., when we apply the decoupling result for the next time, the "new" $A_{1}$ may be inside the "previous" $A_{2}$ )

Remark 1.4. In the course of the proof of the above theorems we actually prove a stronger result: the same conditional decoupling inequality holds true if we replace the sets $\mathcal{I}_{A_{1}}^{u} \subset A_{1}$ and $\mathcal{I}_{A_{3}}^{u} \subset A_{3}$ by sets of random walk excursions in $A_{1}$ and $A_{3}$ (we also have to replace the function $f$ by an increasing function on the set of excursions). That is, the conditional decoupling continues to work when we replace the ranges of the excursions (which constitute the random interlacements set) by the actual excursions themselves. We chose to state the results in the above manner for the sake of clarity and brevity. Note that this remark also applies to the decoupling obtained by Popov and Teixeira (2015).

Remark 1.5. The above theorems can be proved in the same way if we replace the hypercube $A_{1}^{\square}$ by a box $\left[0, a_{1}\right] \times \cdots \times\left[0, a_{d}\right]$, with $c^{-1} r<a_{i}<c r$ for all $i=1, \ldots, d$, 
and some constant $c>1$, and then replace the set $A_{2}^{\square}$ accordingly. We chose to prove the theorems for $A_{1}^{\text {口 }}$ only to simplify the notation. We also note that we prove the theorem for both balls and boxes because the error term obtained in the decoupling for balls is much smaller than the error obtained in the decoupling for boxes, but at the same time the decoupling between boxes tends to be more useful because boxes cover the space in a much more efficient manner.

Remark 1.6. For $d=3$, the only way to obtain an exponentially small (instead of a stretched exponentially small) error term in equations (1.10) and (1.11) is to allow the distance $\sim s$ between the sets $A_{1}$ and $A_{2}$ to be of the same order of the minimal diameter $\sim r$.

Here is an overview of the paper. In Subsection 1.1, we discuss an application of some of our results. Section 2 is devoted to establish the notation and state further definitions. In Section 3 we show how we simulate the interlacements set $\mathcal{I}_{A_{1}}^{u}$ conditioned on the information given by $\mathcal{I}_{A_{2}}^{u}$ using a suitable version of the soft local times method. Finally, in Section 4, we prove the main theorem using a large deviations estimate for the soft local times associated with $\mathcal{I}_{A_{1}}^{u}$. The Appendix is then used to collect and derive the technical estimates we need.

Now, let us comment on how this paper relates to Popov and Teixeira (2015). As observed above, the present results neither imply nor follow from those of Popov and Teixeira (2015). The very main idea of the proof remains the same: construct the processes on the same probability space using the soft local times method, and then prove that one soft local time lies completely above the other with high probability. However, carrying this program out for the conditional decoupling is a much more nontrivial task, for the following reasons:

- There are some fundamental differences in the construction of the conditioned process itself. The unconditional excursion process was a Markov chain, with a lot of regenerations. On the other hand, here, given a specific configuration of the clothesline process defined in (3.7), the excursions of the conditioned process are independent, but not identically distributed (using a RWRE analogy, one may think of the conditioned excursion process as a process in a quenched random environment, where the realization of the random environment is a instance of the aforementioned clothesline process, whose law depends on the interlacements configuration on $A_{3}$ ).

- To control the soft local times, in Popov and Teixeira (2015) one basically only needed to control the entrance measures (given the starting point, the excursion was "free", i.e., just a simple random walk). Here, however, the excursion space has a more complicated base measure $\mu$ (cf. the definitions in Section 3): we have to take both the entrance and exit point into account, and the excursions' law is conditional. There is an additional problem that the estimates on the soft local time increment depend on the relative position of the two points, as well as on the domain's shape itself.

- Due to the above, the task of controlling the first two moments and the tails of soft local times becomes more complicated as well (as the reader may have inferred from the size of this paper). Also, it is probable that the final results are not sharp: we were not able to obtain the $s^{d-2}$-factor in the exponents (as in Popov and Teixeira, 2015), the distance between the sets needs to be polynomial with respect to the diameter, and also the results for balls and boxes are different (as contrasted to those of Popov and 
Teixeira, 2015, where it was possible to obtain the result for fairly general sets).

1.1. An application: biased random walk on the interlacement set. Let $G$ be some (possibly random) subset of $\mathbb{Z}^{d}, d \geq 2$. Fix a parameter $\beta>0$, which accounts for the bias; also, fix some non-zero vector $\ell \in \mathbb{Z}^{d}$. Let us define the conductances on the edges of $\mathbb{Z}^{d}$ in the following way:

$$
\mathcal{C}(x, y)= \begin{cases}e^{\beta(x+y) \cdot \ell}, & \text { if } x, y \text { are neighbors and belong to } G, \\ 0, & \text { otherwise }\end{cases}
$$

and we call the collection of all conductances $\omega=\left\{\mathcal{C}(x, y), x, y \in \mathbb{Z}^{d}\right\}$ the random environment. Consider a random walk $\left(X_{n}, n \geq 0\right)$ in this environment of conductances; i.e., its transition probabilities are given by

$$
P^{\omega}\left[X_{n+1}=y \mid X_{n}=x\right]=\frac{\mathcal{C}(x, y)}{\sum_{z} \mathcal{C}(x, z)}
$$

(the superscript in $P^{\omega}$ indicates that we are dealing with the "quenched" probabilities, i.e., when the underlying random graph / conductancies are already fixed).

There have been significant interest towards this model in recent years, mainly in the case when $G$ is the infinite cluster of supercritical Bernoulli percolation model, see e.g. Berger et al. (2003); Sznitman (2003); Fribergh (2013). In particular, one remarkable fact is the following: the walk is ballistic (transient and with positive speed) in the direction of the drift if $\beta>0$ is small enough; however, it moves only sublinearly fast (its displacement is only of order $t^{a}$ by time $t$ with $a \in(0,1)$, as proved in Fribergh and Hammond, 2014) for large values of $\beta$.

In the work Fribergh and Popov (2018) the case $G=\mathcal{I}^{u}$ was considered. It turned out that in dimension $d=3$, for any value of $\beta>0$, although still transient in the direction of the drift, the walk is not only sub-ballistic, but has also subpolynomial speed, in the sense that its distance to the origin grows slower than $t^{\varepsilon}$ for any $\varepsilon>0$. This is also in contrast with the result that the walk on $\mathcal{I}^{u}$ without any drift is diffusive (so, loosely speaking, its "speed" is $\sqrt{t}$ ), as shown in Procaccia et al. (2016).

We will not describe all the details of Fribergh and Popov (2018) here, but the main idea is the following. As in the case of the biased walk on the infinite percolation cluster, to prove zero speed one needs to show that the walk frequently gets caught in traps. These traps are "dead ends" of the environment looking in the direction of the bias, see Figure 1.2. When the walk enters such a trap, the bias prevents it from goint out, so there is a good chance that the walk will spend quite a lot of time there, and this effectively leads to zero speed. Now, the crucial fact is that, specifically in three dimensions, it is much cheaper to have a trap in the interlacement set than in the (Bernoulli) percolation cluster. Indeed, it is possible to show that the capacity of the dotted set on Figure 1.2 is of order $\frac{\ln t}{\ln \ln t}$ for any fixed $\alpha<1$. The formula (1.1) then shows that having a trap as above has only a subpolynomial (in $t$ ) cost; also, it turns out that "forcing" a trajectory to create a "dead end" as shown on the picture is not too costly as well.

So, when the walk advances in the direction of the bias, from time to time it will encounter a trap and be trapped. However, to make such an argument rigorous, one has to face the following difficulty. When the walk already explored some parts of the environment and then came to an unexplored area, we can no longer use (1.1) 


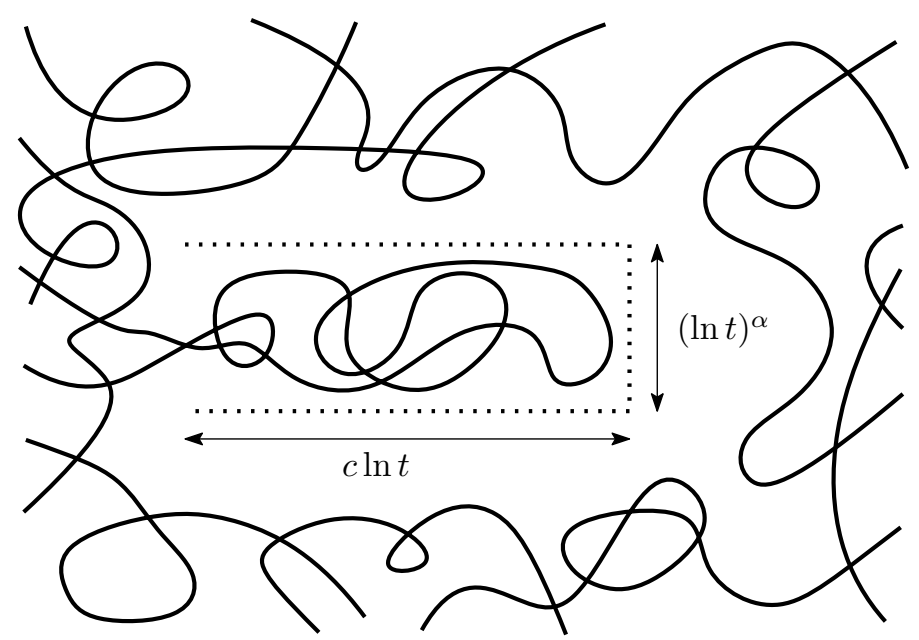

Figure 1.2. A trap for the random walk on the interlacement set (on this picture, the bias is directed along the first coordinate vector). Only the interlacements are shown; the trajectory of the RWRE $X$ is not present on the picture.

to estimate the probability that there is a trap in front of it, due to the lack of independence. It is here that the conditional decoupling enters the scene: it is possible to use the main results of this paper to show that probability of having a trap in front of the particle (when it comes to an unexplored area) is not very small. As mentioned above, the detailed argument can be found in Fribergh and Popov (2018).

\section{Definitions and notation}

In this section we will introduce the basic definitions, conventions and notation used in this paper. We start by stating our convention regarding constants: $c, c^{\prime}$, $c_{1}, c_{2}, c_{3}, \ldots$ are always defined as strictly positive constants depending only on the dimension $d$. Constants can also change value from line to line, unless when the text explicitly states the contrary.

We let $\|\cdot\|$ and $\|\cdot\|_{\infty}$ denote the Euclidean and $\ell_{\infty}$ norms in $\mathbb{Z}^{d}$ respectively. For $x, y \in \mathbb{Z}^{d}$, we also let $\operatorname{dist}(x, y) \equiv\|x-y\|$. We say that two vertices $x, y \in \mathbb{Z}^{d}$ are neighbors when $\|x-y\|=1$, this notion introduces the usual nearest-neighbor graph structure in $\mathbb{Z}^{d}$. For $x \in \mathbb{Z}^{d}$ and $r \in \mathbb{R}_{+}$, we define

$$
B(x, r):=\left\{y \in \mathbb{Z}^{d} ;\|y-x\| \leq r\right\},
$$

the discrete ball in the Euclidean norm centered on $x$ with radius $r$, and

$$
B_{\infty}(x, r):=\left\{y \in \mathbb{Z}^{d} ;\|y-x\|_{\infty} \leq r\right\},
$$

the discrete ball in the $\ell_{\infty}$-norm centered on $x$ with radius $r$. Given a set $A \subseteq \mathbb{Z}^{d}$ we denote by

$$
A^{C}:=\left\{x \in \mathbb{Z}^{d} ; x \notin A\right\}
$$


its complement and by

$$
\partial A:=\left\{x \in A ; \text { there exists } y \in A^{C} \text { such that }\|x-y\|=1\right\}
$$

its (internal) boundary.

For any set $Z$ and any two functions $f, g: Z \rightarrow \mathbb{R}$, we write $f(z) \asymp g(z)$ to denote the fact that there exist two strictly positive constants, $c_{1}$ and $c_{2}$, such that $c_{1} f(z) \leq g(z) \leq c_{2} f(z)$ for all $z \in Z$. When $Z$ is equal to $\mathbb{R}$ we say that $f(z)=o(g(z))$ when $\frac{f(z)}{g(z)}$ goes to 0 as $z \rightarrow \infty$.

Given $x \in \mathbb{Z}^{d}$, we let $\mathbb{P}_{x}$ denote the probability measure associated with the simple random walk in $\mathbb{Z}^{d}$ started at $x$. We will also let $\left(X_{k}, k \geq 0\right)$ denote the simple random walk process in $\mathbb{Z}^{d}$. Given a set $A \subset \mathbb{Z}^{d}$, we define the entrance time for the set $A$

$$
H_{A}:=\inf \left\{k \geq 0 ; X_{k} \in A\right\} .
$$

We also let the hitting time for $A$ be defined as

$$
\tilde{H}_{A}:=\inf \left\{k \geq 1 ; X_{k} \in A\right\} .
$$

When $A$ is finite we denote its harmonic measure by

$$
e_{A}(x)=\mathbb{1}_{x \in A} \mathbb{P}_{x}\left[\tilde{H}_{A}=\infty\right] \text { for } x \in \mathbb{Z}^{d} .
$$

We are then able to define the capacity of the set $A$

$$
\operatorname{cap}(A):=\sum_{x \in A} e_{A}(x),
$$

and the normalized harmonic measure

$$
\bar{e}_{A}(x):=e_{A}(x) \operatorname{cap}(A)^{-1} .
$$

We now write down the definition of the Green function for the simple random walk in $\mathbb{Z}^{d}$ : for $x, y \in \mathbb{Z}^{d}$, we let

$$
G(x, y):=\sum_{k \geq 0} \mathbb{P}_{x}\left[X_{k}=y\right]
$$

Theorem 1.5.4 of Lawler (2013) provides us with the following estimate on the Green function:

$$
G(x, y) \asymp \frac{1}{1+\|x-y\|^{d-2}} .
$$

An application of the optional stopping theorem then gives us the following elementary result:

Lemma 2.1. Let $0<\rho_{1}<\rho_{2}$ be sufficiently large real numbers, and let $x \in$ $B\left(0, \rho_{2}\right) \backslash B\left(0, \rho_{1}\right)$. Then

$$
\mathbb{P}_{x}\left[H_{\partial B\left(0, \rho_{1}\right)}<H_{\partial B\left(0, \rho_{2}\right)}\right]=\frac{|x|^{-(d-2)}-\left(\rho_{2}\right)^{-(d-2)}+O\left(\left(\rho_{1}\right)^{-(d-1)}\right)}{\left(\rho_{1}\right)^{-(d-2)}-\left(\rho_{2}\right)^{-(d-2)}} .
$$

Let us briefly discuss the definition of the measure associated with the random interlacements process intersected with a given finite set $A \subset \mathbb{Z}^{d}$. Assume we have constructed a probability space where, for every $i \geq 1$, there exists a simple random walk process $\left(X_{k}^{(i)}, k \geq 0\right)$ with starting distribution given by $\bar{e}_{A}(\cdot)$, and such that $\left(X_{k}^{(i)}, k \geq 0\right)$ is independent from $\left(X_{k}^{(j)}, k \geq 0\right)$ for $i \neq j$. We also assume that in this space we can construct an independent Poisson process $\left(J_{u}\right)_{u \geq 0}$ on the positive 
real line with intensity $u \cdot \operatorname{cap}(A)$. The law of the random interlacements process $\left(\mathcal{I}^{u}\right)_{u \geq 0}$ intersected with the set $A$ can then be characterized by

$$
\left(\mathcal{I}^{u} \cap A\right)_{u \geq 0} \stackrel{d}{=}\left(A \cap \bigcup_{i \leq J_{u}} \bigcup_{k \geq 0} X_{k}^{(i)}\right)_{u \geq 0}
$$

as can be seen in Sznitman (2010), Proposition 1.3, or in the paragraph before (2.6) in Černý and Teixeira (2016). This definition gives rise to compatible measures in the following sense: Given two finite sets $K_{1} \subset K_{2} \subset \mathbb{Z}^{d}$, we have that $\left(\left(\mathcal{I}^{u} \cap\right.\right.$ $\left.\left.K_{2}\right)_{u \geq 0}\right) \cap K_{1}$ has the same law as $\left(\mathcal{I}^{u} \cap K_{1}\right)_{u \geq 0}$.

We now introduce the concept of the "smoothed hypercube". Throughout this paper we will need to use estimates on the hitting measure of a simple random walk on the sets $A_{1}$ and $A_{2}$. However, the sharp edges present on $A_{1}^{\square}$ and $A_{2}^{\square}$ greatly complicate such estimates. Instead we will prove our main results in the context of "smoothed" versions of these sets, that is, we let

$A_{1}^{\square}:=\left\{x \in \mathbb{Z}^{d} ; B(x, s) \subset B_{\infty}(0, r)\right\}, A_{2}^{\square}:=\left(\left\{x \in \mathbb{Z}^{d} ; B(x, s) \subset B_{\infty}(0, r+2 s)\right\}\right)^{C}$.

We will later explain how to obtain our main results for the unsmoothed versions of these sets.

To finish the section, we define $V^{\circ}:=V^{\bigcirc}(r, s)$ to be the boundary set

$$
V^{\circ}:=\partial\left\{x_{1} \in \mathbb{Z}^{d}, \operatorname{dist}\left(x_{1}, x_{2}\right) \leq s \text { for some } x_{2} \in A_{1}^{\circ}\right\},
$$

and we define $V^{\square}:=V^{\square}(r, s)$ in an analogous way. Since $s=o(r)$, we have

$$
\operatorname{cap}(V)=\operatorname{cap}\left(A_{2}^{C}\right)(1+o(1))=\operatorname{cap}\left(A_{1}\right)(1+o(1)),
$$

and also, by Proposition 2.2.1 and equation (2.16) of Lawler (2013),

$$
\operatorname{cap}(V) \asymp r^{d-2} .
$$

\section{Simulating excursions with soft local times}

We begin the present section with a short recapitulation of the technique introduced in Popov and Teixeira (2015), the Soft Local Times method. This review will also be important in order to reformulate the results of Popov and Teixeira (2015) in a more general setting (the original paper was written with a Markovian setting in mind).

The method essentially allows us to simulate any number of random variables taking values in a state space $\Sigma$ using a realization of a Poisson point process in $\Sigma \times \mathbb{R}_{+}$.

Let $\Sigma$ be a locally compact Polish metric space, and let $\mathcal{B}(\Sigma)$ be its Borel $\sigma$ algebra. Let $\mu$ be a Radon measure over $\mathcal{B}(\Sigma)$, so that every compact set has finite $\mu$-measure.

Such measure space $(\Sigma, \mathcal{B}(\Sigma), \mu)$ is the usual setup for the construction of a Poisson point process on $\Sigma$. We consider the space of Radon point measures in $\Sigma \times$ $\mathbb{R}_{+}$

$$
L=\left\{\eta=\sum_{\lambda \in \Lambda} \delta_{\left(z_{\lambda}, v_{\lambda}\right)} ; z_{\lambda} \in \Sigma, v_{\lambda} \in \mathbb{R}_{+} \text {and } \eta(K)<\infty \text { for all compact } K\right\},
$$

endowed with the $\sigma$-algebra $\mathcal{D}$ generated by the evaluation maps

$$
\eta \mapsto \eta(D), \quad D \in \mathcal{B}\left(\mathbb{R}_{+}\right) \otimes \mathcal{B}(\Sigma) .
$$


We are then able to construct a Poisson point process $\eta$ in the space $(L, \mathcal{D}, \mathbb{Q})$ with intensity measure given by $\mu \otimes \mathrm{d} v$, where $\mathrm{d} v$ is the Lebesgue measure on $\mathbb{R}_{+}$, see Resnick (2008), Proposition 3.6 on p.130.

Let $X_{1}, X_{2}, \ldots, X_{n}$ be random variables on $\Sigma$ such that $X_{1}$ 's distribution is absolutely continuous with respect to $\mu$ and, for all $i=2, \ldots, n$, the law of $X_{i}$ conditioned on the values taken by $X_{1}, \ldots, X_{i-1}$ is absolutely continuous with respect to $\mu$. Using the process $\eta$ constructed above, we define

$$
\begin{aligned}
& g_{1}: \Sigma \mapsto \mathbb{R}_{+}, \text {the density function of } X_{1} \text { with respect to } \mu, \\
& \xi_{1}:=\inf \left\{t \geq 0 ; \text { there exists } \lambda \in \Lambda \text { such that } g_{1}\left(z_{\lambda}\right) \geq v_{\lambda}\right\}, \\
& G_{1}(z):=\xi_{1} g_{1}(z), \text { for } z \in \Sigma \\
& \left(z_{\lambda_{1}}, v_{\lambda_{1}}\right), \text { the unique pair in }\left\{\left(z_{\lambda}, v_{\lambda}\right)\right\}_{\lambda \in \Lambda} \text { with } G_{1}\left(z_{\lambda_{1}}\right)=v_{\lambda_{1}} .
\end{aligned}
$$

Then, recursively, for $2 \leq k \leq n$ we define $g_{k}: \Sigma \mapsto \mathbb{R}_{+}$to be the density function of $X_{k}$ conditioned on the event $\left\{X_{1}=z_{\lambda_{1}}, \ldots, X_{k-1}=z_{\lambda_{k-1}}\right\}$,

$$
\begin{aligned}
& \xi_{k}:=\inf \left\{t \geq 0 ; \text { there exists } \lambda \in \Lambda \text { such that } \operatorname{tg}_{k}\left(z_{\lambda}\right)+G_{k-1}\left(z_{\lambda}\right) \geq v_{\lambda}\right\}, \\
& G_{k}(z):=\xi_{k} g_{k}(z)+G_{k-1}(z), \text { for } z \in \Sigma \\
& \left(z_{\lambda_{k}}, v_{\lambda_{k}}\right) \text {, the unique pair in }\left\{\left(z_{\lambda}, v_{\lambda}\right)\right\}_{\lambda \in \Lambda} \text { with } G_{k}\left(z_{\lambda_{k}}\right)=v_{\lambda_{k}} .
\end{aligned}
$$

Using Proposition 4.1 of Popov and Teixeira (2015), we are able to state the following proposition:

Proposition 3.1. The vector $\left(z_{\lambda_{1}}, \ldots, z_{\lambda_{n}}\right)$ has the same law as $\left(X_{1}, \ldots, X_{n}\right)$.

We call the function $G_{n}(z)$ the soft local time of the vector $\left(X_{1}, \ldots, X_{n}\right)$ up to time $n$ with respect to the measure $\mu$, or more usually simply the soft local time. If $T$ is a stopping time with respect to the canonical filtration generated by the variables $X_{i}$, it is simple to define $G_{T}(z)$, the soft local time up to time $T$.

Note that by controlling the value of the soft local times function we will automatically control the values our random variables take, as the next corollary summarizes:

Corollary 3.2. For any measurable function $h: \Sigma \rightarrow \mathbb{R}_{+}$we have, using the same notation as above,

$$
\mathbb{Q}\left[\left\{z_{1}, \ldots, z_{T}\right\} \subseteq\left\{z_{\lambda} ; v_{\lambda} \leq h\left(z_{\lambda}\right)\right\}\right] \geq \mathbb{Q}\left[G_{T}(z) \leq h(z), \text { for } \mu \text {-a.e. } z \in \Sigma\right],
$$

for any finite stopping time $T \geq 1$.

We will now use the above result in our random interlacements context. More specifically, we will show a way of simulating the intersection of the random interlacements set with a given subset of $\mathbb{Z}^{d}$ in such a way as to make explicit the dependence each random walk excursion has with its entrance and exit points on the subset. We refer the reader to Figure 3.3 for a brief overview of the arguments used in this section.

It is clear from (2.3) that in order to simulate the random interlacements set at level $u$ in a bounded subset $K$ of $\mathbb{Z}^{d}$ we need only to pick $N_{K}^{u} \stackrel{d}{=} \operatorname{Poisson}(u \operatorname{cap}(K))$ points in $\partial K$, each point chosen according to the measure $\bar{e}_{K}(\cdot)$, and from each point start a simple random walk.

We intend to study $\mathcal{I}_{A_{1}}^{u}=\mathcal{I}^{u} \cap A_{1}$, showing that this set is not that much influenced by the random interlacements set intersected with $A_{2}, \mathcal{I}_{A_{2}}^{u}=\mathcal{I}^{u} \cap A_{2}$. 


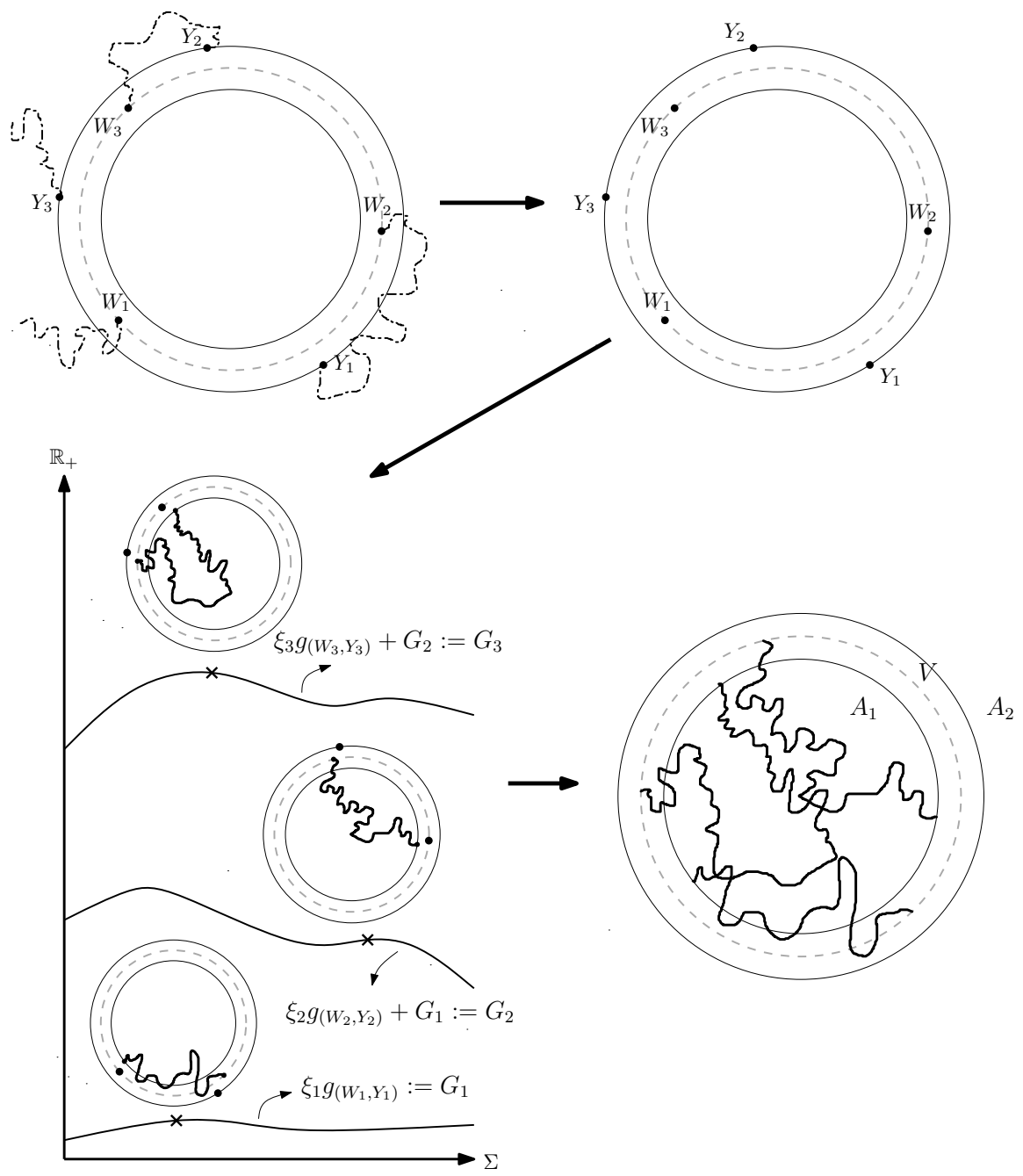

Figure 3.3. The figure shows how we use the soft local times technique to simulate the range of a simple random walk trajectory intersected with $A_{1}$. We first simulate a process of pairs of points $\left(\left(W_{k}, Y_{k}\right), k \geq 0\right)$ denoting the entrance at $V$ and exit at $\partial A_{2}$ of a simple random walk trajectory that starts at $V$. We then use the soft local times method to simulate the pieces of trajectory that lie between each of the pairs $\left(W_{k}, Y_{k}\right)$.

We will later clarify what we mean by "influence". For now, we observe that the only "information" $\mathcal{I}_{A_{1}}^{u}$ receives from $\mathcal{I}_{A_{2}}^{u}$ is the location of the entrance and exit points of the excursions on $\partial A_{2}$ of the random walks that constitute $\mathcal{I}_{A_{2}}^{u}$.

Let us now describe how the process is constructed. We first generate the points of entrance at $V$ and exit from $A_{2}^{C}$ of each excursion on $V$ of a random walk trajectory. These points will be the clothesline onto which we will hang the pieces of trajectory that meet $A_{1}$, we will do so using the soft local times method. 
Let us define the successive return and departure times between $V$ and $A_{2}$. Given a trajectory that starts at $V$, we define

$$
\begin{array}{llrl}
D_{0}=0, & & R_{1}=H_{\partial A_{2}}, \\
D_{1}=H_{V} \circ \theta_{R_{1}}+R_{1}, & & R_{2}=H_{\partial A_{2}} \circ \theta_{D_{1}}+D_{1}, \\
D_{2}=H_{V} \circ \theta_{R_{2}}+R_{2} & & \text { and so on. }
\end{array}
$$

We also define the random time

$$
T_{\Delta}=\inf \left\{k \geq 1 ; R_{k}=\infty\right\},
$$

which is almost surely finite, as the walk is transient.

Let $\left(X_{n}, n \geq 0\right)$ be the simple random walk with initial distribution given by $\bar{e}_{V}(\cdot)$. Let $\Delta$ be an artificial cemetery state. We construct a random sequence of elements of $\left(V \times \partial A_{2}\right) \cup\{\Delta\}$ in the following way: Conditioned on the event $\left\{T_{\Delta}=m\right\}$, we let

$$
\begin{aligned}
\left(\left(W_{1}, Y_{1}\right), \ldots,\left(W_{m-1}, Y_{m-1}\right),\left(W_{m}, Y_{m}\right),\left(W_{m+1}, Y_{m+1}\right), \ldots\right) & \\
= & \left(\left(X_{D_{0}}, X_{R_{1}}\right), \ldots,\left(X_{D_{m-2}}, X_{R_{m-1}}\right), \Delta, \Delta, \ldots\right) .
\end{aligned}
$$

It is then elementary to prove that the process $\left(\left(W_{k}, Y_{k}\right)\right)_{k \geq 1}$ inherits the Markov property from the simple random walk. We call $\left(\left(W_{k}, Y_{k}\right)\right)_{k \geq 1}$ the clothesline process started at $W_{1}$. When there is no risk of confusion we will also denote by $\mathbb{P}_{w_{0}}$ the probability measure associated with the clothesline process started at a given point $w_{0} \in V$.

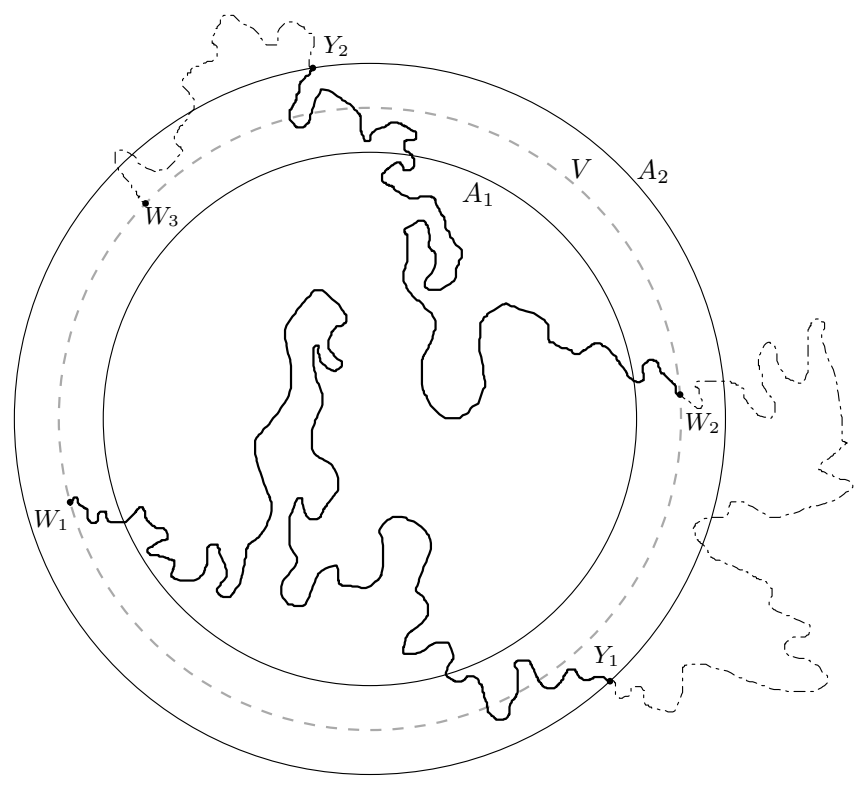

Figure 3.4. An example of the process $\left(\left(W_{k}, Y_{k}\right)\right)_{k \geq 1}$.

Let us now use the soft local times method to generate the trajectories inside $A_{1}$, given the entrance and exit points $\left(\left(W_{k}, Y_{k}\right)\right)_{k \geq 1}$. We first define the underlying 
space $\Sigma$ where our pieces of trajectories will live. We let $\mathcal{K}$ be the set of nearestneighbor paths in $A_{2}^{C}$ with one endpoint in $\partial A_{1}$ and the other in $V$,

$$
\mathcal{K}:=\left\{\left(x_{0}, x_{1}, \ldots, x_{n}\right) ; n \in \mathbb{N}, x_{i} \in A_{2}^{C} \text { for } 1 \leq i \leq n, x_{0} \in \partial A_{1}, x_{n} \in V\right\} .
$$

We introduce yet another artificial state $\Theta$ for reasons that will be made clear in

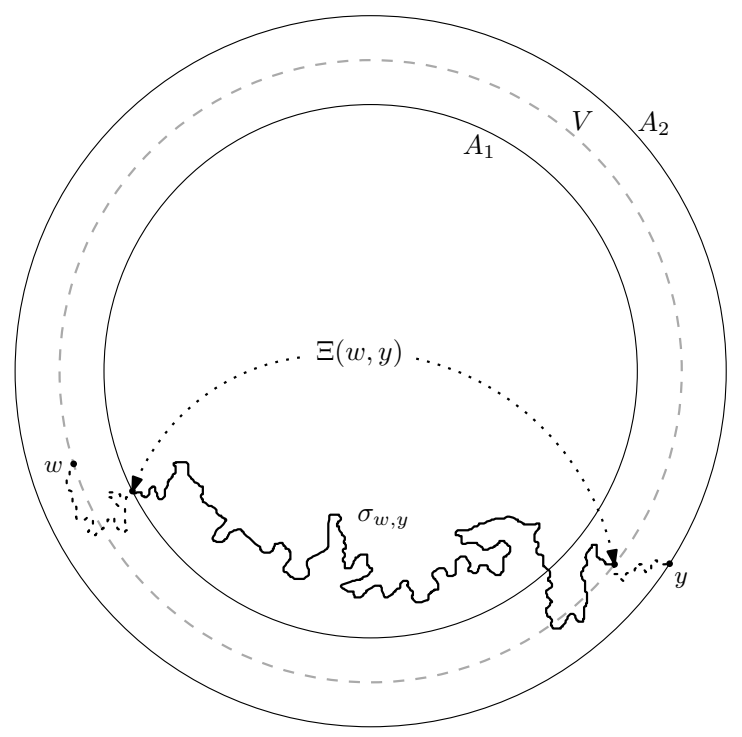

FiguRE 3.5. The definition of $\sigma(w, y)$ and $\Xi(w, y)$.

a few moments. We let $\Sigma:=\mathcal{K} \cup\{\Theta\}$ and let $\mu$ be a measure on $\Sigma$ defined in the following way: given $A \subseteq \Sigma$,

$$
\mu(A):=\sum_{\left(x_{0}, \ldots, x_{n}\right) \in A} \tilde{\mathbb{P}}_{\left(x_{0}, x_{n}\right)}\left[X_{0}=x_{0}, \ldots, X_{n}=x_{n}\right]+1_{\{\Theta \in A\}},
$$

where $\tilde{\mathbb{P}}_{\left(x_{0}, x_{n}\right)}$ is the simple random walk measure conditioned on the event where $x_{0}$ is the walk's initial point and $x_{n}$ is its last point on $V$ before reaching $\partial A_{2}$. Notice that $\mu(\{\Theta\})=1$.

Given $(w, y) \in V \times \partial A_{2}$ we let $\hat{\mathbb{P}}_{w, y}$ be the measure associated with simple random walk starting at $w$ conditioned on the event where $y$ is the first point the walk hits in $\partial A_{2}$, that is:

$$
\hat{\mathbb{P}}_{w, y}[\cdot]:=\mathbb{P}_{w}\left[\cdot \mid X_{H_{\partial A_{2}}}=y\right]
$$

We want to randomly select (according to the conditional simple random walk measure above) a piece of trajectory in $A_{1}$ given a starting point in $V$ and an ending point in $\partial A_{2}$. Given $w \in V$ and $y \in \partial A_{2}$ we define the random element $\sigma_{w, y} \in \Sigma$ in the following way:

- Let $\mathcal{B}_{w, y}$ be a Bernoulli random variable with parameter $\hat{\mathbb{P}}_{w, y}\left[H_{\partial A_{1}}<\right.$ $\left.H_{\partial A_{2}}\right]$.

- If $\mathcal{B}_{w, y}=0$ we let $\sigma_{w, y} \equiv \Theta$. 
- If $\mathcal{B}_{w, y}=1$ we let, for $\mathfrak{A} \subseteq \mathcal{K}$ :

$$
\mathbb{P}\left[\sigma_{w, y} \in \mathfrak{A}\right]=\sum_{\left(a_{0}, \ldots, a_{n}\right) \in \mathfrak{A}} \hat{\mathbb{P}}_{w, y}\left[\begin{array}{c}
X_{H_{A_{1}}}=a_{0}, X_{H_{A_{1}}+1}=a_{1}, \ldots, X_{H_{A_{1}}+n}=a_{n}, \\
X_{k} \notin V \text { for every } k=H_{A_{1}}+n+1, \ldots, H_{A_{2}}
\end{array}\right]
$$

In other words, the random element $\sigma_{w, y} \in \Sigma$ will either be $\Theta$, on the event where a random walk starting at $w$ and exiting at $y$ fails to reach $A_{1}$, or a simple random walk trajectory $\left(x_{0}, x_{1}, \ldots, x_{k}\right) \in \mathcal{K}$ distributed so that $x_{0}$ is the first point in $A_{1}$ after the start at $w$ and $x_{k}$ is the last point in $V$ before reaching $y \in \partial A_{2}$. We then define $g_{(w, y)}: \Sigma \mapsto \mathbb{R}_{+}$to be the $\mu$-density of $\sigma_{w, y}$. We refer to Figure 3.5.

Given $z=\left(x_{0}, \ldots, x_{n}\right) \in \mathcal{K}$ we denote by $\Xi(z)$ the pair $\left(x_{0}, x_{n}\right)$, the path's starting and ending points. We also let $\Xi(\Theta)=\Theta$ so that $\Xi(z)$ is defined for all $z \in \Sigma$. For $(w, y) \in V \times \partial A_{2}$ we define $\Xi(w, y)$ to be the random element $\Xi\left(\sigma_{w, y}\right)$.

Let us calculate $g_{(w, y)}$ using the above notation. For $\mathfrak{A} \subseteq \Sigma$ we want to express the probability $\mathbb{P}\left[\sigma_{w, y} \in \mathfrak{A}\right]$ as a $\mu$-integral over $\mathfrak{A}$.

$$
\begin{aligned}
\mathbb{P}\left[\sigma_{w, y} \in \mathfrak{A}\right]= & \sum_{a \in \mathfrak{A}} \mathbb{P}\left[\sigma_{w, y}=a\right] \\
= & 1_{\{\Theta \in \mathfrak{A}\}} \hat{\mathbb{P}}_{w, y}[\Xi(w, y)=\Theta] \\
& +\sum_{\substack{a \in \mathfrak{A} \\
a \neq \Theta}} \hat{\mathbb{P}}_{w, y}[\Xi(w, y)=\Xi(a)] \hat{\mathbb{P}}_{w, y}[a \mid \Xi(w, y)=\Xi(a)] \\
= & 1_{\{\Theta \in \mathfrak{A}\}} \hat{\mathbb{P}}_{w, y}[\Xi(w, y)=\Theta]+\sum_{\substack{a \in \mathfrak{A} \\
a \neq \Theta}} \hat{\mathbb{P}}_{w, y}[\Xi(w, y)=\Xi(a)] \tilde{\mathbb{P}}_{\Xi(a)}[a] \\
= & \sum_{a \in \mathfrak{A}} \hat{\mathbb{P}}_{w, y}[\Xi(w, y)=\Xi(a)] \mu(a) \\
= & \int_{\mathfrak{A}} \hat{\mathbb{P}}_{w, y}[\Xi(w, y)=\Xi(z)] \mu(\mathrm{d} z),
\end{aligned}
$$

so that $g_{(w, y)}(z)=\hat{\mathbb{P}}_{w, y}[\Xi(w, y)=\Xi(z)]$. Notice that the function $g_{(w, y)}(z)$ only depends on the pair $\Xi(z)$, the path's initial and ending points.

Let $(L, \mathcal{D}, \mathbb{Q})$ be the measure space of the Poisson point process on $\Sigma \times \mathbb{R}_{+}$with intensity measure $\mu \otimes \mathrm{d} v$, where $\mathrm{d} v$ is the Lebesgue measure on $\mathbb{R}_{+}$. A weighted sum of functions $g_{(\cdot, \cdot)}$ indexed by clothesline processes $\left(\left(W_{k}, Y_{k}\right)\right)_{k \geq 1}$ will be the soft local time used to simulate the pieces of trajectory we need. This way we will be able to simulate the intersection of a simple random walk trajectory with $A_{1}$. As we have seen in the random interlacements process's definition, to simulate the interlacements set inside $V$ we need a number $N_{V}^{u} \stackrel{d}{=} \operatorname{Poisson}(u \operatorname{cap}(V))$ of independent random walks. We will need the same number of independent clothesline processes. For such task we will need a much bigger probability space, easily definable as a product between the Poisson point process space and an infinite product of independent simple random walk spaces starting on $V$. We call this bigger space the global probability space, and denote by $\mathcal{P}$ its distribution. Abusing the notation slightly, we will also write $\mathbb{E}$ to denote the expectation associated with this probability space. 

time:

Given a clothesline process $\left(\left(W_{k}, Y_{k}\right)\right)_{k \geq 1}$, we define the trajectory's soft local

$$
G(z)=\sum_{k=1}^{T_{\Delta}} \xi_{k} g_{\left(W_{k}, Y_{k}\right)}(z) .
$$

We will also need to consider the soft local time up to a random time $T \leq T_{\Delta}$ :

$$
G_{T}(z)=\sum_{k=1}^{T} \xi_{k} g_{\left(W_{k}, Y_{k}\right)}(z)
$$

Analogously, we define for any deterministic time $n \geq 1$

$$
G_{n}(z)=\sum_{k=1}^{n} \xi_{k} g_{\left(W_{k}, Y_{k}\right)}(z)
$$

We denote by $z_{k}$ the piece of trajectory randomly selected by the $k$-th soft local time, $G_{k}$.

As we have seen before, in order to simulate the random interlacements set at level $u$ in $A_{1}$, we actually need a

$$
N_{V}^{u} \stackrel{d}{=} \operatorname{Poisson}(u \operatorname{cap}(V))
$$

number of random walk trajectories, each started at a point in $V$ distributed as $\bar{e}_{V}(\cdot)$. For $j=1, \ldots, N_{V}^{u}$ we let $\left(\left(W_{k}^{j}, Y_{k}^{j}\right)\right)_{k \geq 1}$ be a clothesline process started at $W_{1}^{j}$, so that $\left(\left(W_{k}^{j}, Y_{k}^{j}\right)\right)_{k \geq 1}$ is independent from $\left(\left(W_{k}^{i}, Y_{k}^{i}\right)\right)_{k \geq 1}$ for $i \neq j$, and so that $W_{1}^{j}$ is distributed as $\bar{e}_{V}(\cdot)$. Let $T_{\Delta}^{j}$ be the killing time associated with $\left(\left(W_{k}^{j}, Y_{k}^{j}\right)\right)_{k \geq 1}$. We denote by

$$
G^{j}(z)=\sum_{k=1}^{T_{\Delta}^{j}} \xi_{k}^{j} g_{\left(W_{k}^{j}, Y_{k}^{j}\right)}(z)
$$

the soft local time associated with the $j$-th clothesline process. It should be clear from Proposition 3.1 that we can simulate all the random elements $\left(\sigma_{W_{k}^{j}, Y_{k}^{j}}\right)_{j, k \geq 1}$ at the same time using only one realization of a Poisson point process in $\Sigma \times \mathbb{R}_{+}$. As the Corollary 3.2 shows, in order to control the values our random elements take we only need to control the function

$$
G_{u}^{\Sigma}(z)=\sum_{j=1}^{N_{V}^{u}} G^{j}(z)
$$

the soft local time associated with the whole process. With such objective in mind we for now set our goals at estimating the soft local time's moments. We first show an easier way to express the expectation of $G(z)$.

Proposition 3.3. Using the same notation as above, we have

$$
\mathbb{E}(G(z))=\mathbb{E}\left(\sum_{k=1}^{T_{\Delta}} 1_{\left\{\Xi\left(X_{D_{k-1}}, X_{R_{k}}\right)=\Xi(z)\right\}}\right) .
$$


Proof: In fact,

$$
\begin{aligned}
\mathbb{E}(G(z)) & =\mathbb{E}\left(\sum_{k=1}^{T_{\Delta}} g_{\left(W_{k}, Y_{k}\right)}(z)\right)=\mathbb{E}\left(\sum_{k=1}^{T_{\Delta}} \hat{\mathbb{P}}_{W_{k}, Y_{k}}\left[\Xi\left(W_{k}, Y_{k}\right)=\Xi(z)\right]\right) \\
& =\mathbb{E}\left(\sum_{k=1}^{T_{\Delta}} 1_{\left\{\Xi\left(W_{k}, Y_{k}\right)=\Xi(z)\right\}}\right)=\mathbb{E}\left(\sum_{k=1}^{T_{\Delta}} 1_{\left\{\Xi\left(X_{D_{k-1}}, X_{R_{k}}\right)=\Xi(z)\right\}}\right) .
\end{aligned}
$$

We have shown that the expectation of $G(z)$, for $z \neq \Theta$, is the same as the expectation of how many times a random walk started at $W_{1}$ will do an excursion on $A_{2}^{C}$ with starting and ending points given by $\Xi(z)$.

The next proposition gives a bound on the second moment $\mathbb{E}\left(G(z)^{2}\right)$. It is a straightforward adaptation of Theorem 4.8 of Popov and Teixeira (2015) to our context and therefore we omit its proof. In what follows, we let $\mathbb{E}_{w}(G(z))$ denote the expectation of the soft local time $G(z)$ when the associated clothesline process is conditioned on starting at $w \in V$.

Proposition 3.4. For any $w_{0} \in V$,

$$
\mathbb{E}_{w_{0}}\left(G(z)^{2}\right) \leq 2 \mathbb{E}_{w_{0}}(G(z))\left(\sup _{w^{\prime} \in V} \mathbb{E}_{w^{\prime}} G(z)+\sup _{w, y} g_{(w, y)}(z)\right) .
$$

For this paper's results, an estimate on the exponential moments of $G$ will be essential. The next proposition, again adapted from Popov and Teixeira (2015) (Propositions 3.5 and 3.4 are proved in the context of Markov chains in the original paper), gives us such an estimate. Since the proof of the following result is essentially the same as the proof of Theorem 4.9 of Popov and Teixeira (2015), we will omit it.

Proposition 3.5. Given $\hat{z} \in \Sigma$ and a measurable set $\Gamma \subset \Sigma$, let

$$
\begin{aligned}
& \qquad \ell \geq \sup _{(w, y) \in V \times \partial A_{2}} g_{(w, y)}(\hat{z}) . \\
& \text { Then, for any } v \geq 2, \quad \\
& \mathcal{P}[G(\hat{z}) \geq v \ell] \\
& \quad \leq \mathcal{P}[G(\hat{z}) \geq \ell]\left(\exp \left\{-\left(\frac{v}{2}-1\right)\right\}+\sup _{w^{\prime}} \mathcal{P}_{w^{\prime}}\left[\eta\left(\Gamma \times\left[0, \frac{1}{2} v \ell \alpha\right]\right) \leq N(\Gamma)\right]\right)
\end{aligned}
$$$$
\alpha=\inf \left\{\frac{g_{(w, y)}\left(z^{\prime}\right)}{g_{(w, y)}(\hat{z})} ;(w, y) \in V \times \partial A_{2}, z^{\prime} \in \Gamma\right\},
$$$$
N(\Gamma)=\#\left\{k \leq T_{\Delta} ; z_{k} \in \Gamma\right\} \text {, and }
$$

(note that $\eta\left(\Gamma \times\left[0, \frac{1}{2} v \ell \alpha\right]\right)$ is a random variable with distribution $\left.\operatorname{Poisson}\left(\frac{1}{2} v \ell \alpha \mu(\Gamma)\right)\right)$.

\section{Conditional decoupling}

We begin with an overview of the main argument presented in this section. We will sample the random interlacements set intersected with $A_{1}$ in two ways. In the first way we will sample $\mathcal{I}_{A_{1}}^{u}$ using $G_{u}^{\Sigma}$, that is, we will sample $\mathcal{I}_{A_{1}}^{u}$ using the soft local times indexed by a collection of independent clothesline processes. In the second way, we will construct a set made up from random walk trajectories in $A_{1}$ in a similar way to the construction of $\mathcal{I}_{A_{1}}^{u}$, the only difference will be that the soft 
local times used in this second construction will be indexed by a given nonrandom sequence $\hat{\zeta}$ of pairs of points belonging $V \times \partial A_{2}$. We will denote this second random set by $\mathcal{I}_{A_{1} \mid \hat{\zeta}}^{u}$, and we will show using the soft local times method that $\mathcal{I}_{A_{1} \mid \hat{\zeta}}^{u}$ and $\mathcal{I}_{A_{1}}^{u}$ are usually very similar to each other. We then prove a similar result when the pairs of points that constitute the nonrandom sequence all belong to the boundary of a set contained in $A_{2}$.

Throughout this section we will again only differentiate between $A_{1}^{\bigcirc}$ and $A_{1}^{\square}$ when the need arises.

For $\hat{u}>0, \delta \in(0,1)$ and $z \in \mathcal{K}$, we define the interval

$$
I_{\hat{u}, z}^{\delta}:=[(1-\delta) \hat{u} \operatorname{cap}(V) \mathbb{E}[G(z)],(1+\delta) \hat{u} \operatorname{cap}(V) \mathbb{E}[G(z)]]
$$

and the event

$$
D_{\hat{u}}^{\delta}:=\left\{G_{\hat{u}}^{\Sigma}(z) \in I_{\hat{u}, z}^{\delta} \text { for all } z \in \mathcal{K}\right\} .
$$

The main technical goal of this section is to prove the following result:

Proposition 4.1. Recall the definition of $a_{\odot}, a_{\square}$. Using the notation above defined, we have that, for $u>0$ and $\varepsilon \in(0,1 / 4)$, there exist constants $c, c^{\prime}>0$ depending only on the dimension and on whether of the set $A_{1}$ is an Euclidean ball or a hypercube such that

$$
\mathcal{P}\left[D_{u}^{\varepsilon / 4}, D_{u(1-\varepsilon)}^{\varepsilon / 4}, D_{u(1+\varepsilon)}^{\varepsilon / 4}\right] \geq 1-c \exp \left(-c^{\prime} \varepsilon^{2} u s^{a}\right) .
$$

We will prove the above proposition later, after establishing some preliminary results about large deviations for the soft local times we defined. We will first show how we obtain the Theorems 1.1 and 1.2 from (4.1).

Proposition 4.1 implies that each of the soft local times associated to the processes $\mathcal{I}_{A_{1}}^{u}, \mathcal{I}_{A_{1}}^{u(1-\varepsilon)}$ and $\mathcal{I}_{A_{1}}^{u(1+\varepsilon)}$ stay confined between the graphs of two explicit deterministic functions (as can partially be seen in Figure 4.6). This happens when we let the points of entrance at $V$ and exit at $\partial A_{2}$ of the excursions on $A_{1}$ of the simple random walk trajectories of the interlacements process at level $u$ be distributed according to the "right" law, that is, the law of the clothesline processes. That is, when we "average" these points according to this law we obtain a good concentration for the whole function $G_{u}^{\Sigma}$. Nevertheless, our goal is to obtain a similar concentration when these points are deterministic. The heuristic argument is that when something happens with high probability in the "annealed" law, then most of the times it will also happen with high probability in the "quenched" law. Therefore, for "many" of these deterministic entrance and exit points, we will also have a strong concentration inequality result similar to Proposition 4.1. We will introduce some new notation to make this argument rigorous and prove our main theorem.

We let

$$
\left(\mathcal{C}_{u}\left(V, \partial A_{2}\right), \mathcal{B}_{u}\left(V, \partial A_{2}\right), \mathbb{P}_{V, \partial A_{2}}^{u}\right)
$$

be a probability subspace in which the collection of clothesline processes $\left\{\left(W_{k}^{j}, Y_{k}^{j}\right)_{k=1}^{T_{\Delta}^{j}}\right\}_{j=1}^{N_{u}^{V}}$ is defined, where each $\hat{\zeta} \in \mathcal{C}_{u}\left(V, \partial A_{2}\right)$ can be written as a finite collection of finite sequences of points belonging to $V$ and $\partial A_{2}$ :

$$
\hat{\zeta}:=\left\{\hat{\zeta}_{1}, \ldots, \hat{\zeta}_{K}\right\},
$$


where for each $j=1, \ldots, K ; \hat{\zeta}_{j}$ is a finite sequence alternating between points of $V$ and $\partial A_{2}$. We write

$$
\hat{\zeta}_{j}=\left(\zeta_{0}^{j}, \ldots, \zeta_{n(j)}^{j}\right),
$$

where $n(j)$ is odd, every even entry belongs to $V$ and every odd entry belongs to $\partial A_{2}$.

We then define the soft local times associated with $\hat{\zeta}$. Using the same realization of the Poisson point process on $\Sigma \times \mathbb{R}_{+}$defined in Section 3, we construct the soft local times

$$
G^{\hat{\zeta}_{j}}(z):=\sum_{k=0}^{\frac{n(j)-1}{2}} \tilde{\xi}_{k}^{j} g_{\left(\zeta_{2 k}^{j}, \zeta_{2 k+1}^{j}\right)}(z),
$$

where $\tilde{\xi}_{k}^{j}$ is an exponential random variable defined in the manner of (3.13). We then define

$$
G^{\hat{\zeta}}(z):=\sum_{j=1}^{K} G^{\hat{\zeta}_{j}}(z) .
$$

This function should be viewed as a quenched version of the soft local times $G_{u}^{\Sigma}$, when the collection of clothesline processes $\left\{\left(W_{k}^{j}, Y_{k}^{j}\right)_{k=1}^{T_{\Delta}^{j}}\right\}_{j=1}^{N_{u}^{V}}$ is given by the deterministic element $\hat{\zeta}$. We denote by $\mathcal{I}_{A_{1} \mid \hat{\zeta}}^{u}$ the interlacements process inside $A_{1}$ determined by the ranges of the excursions of $\Sigma$ bellow $G^{\hat{\zeta}} . \mathcal{I}_{A_{1} \mid \hat{\zeta}}^{u}$ is distributed as the random interlacements process inside $A_{1}$ when its associated random walks excursions have entrance points at $V$ and exit points at $\partial A_{2}$ given by $\hat{\zeta}$. The next proposition implies that $G^{\hat{\zeta}}$ is usually between $G_{u(1-\varepsilon)}^{\Sigma}$ and $G_{u(1+\varepsilon)}^{\Sigma}$ with high probability.

Proposition 4.2. There exists a set $\mathcal{A} \in \mathcal{B}_{u}\left(V, \partial A_{2}\right)$ such that

$$
\mathbb{P}_{V, \partial A_{2}}^{u}[\mathcal{A}] \geq 1-\exp \left(-\frac{c^{\prime}}{2} \varepsilon^{2} u s^{a}\right),
$$

and for all fixed $\hat{\zeta} \in \mathcal{A}$,

$$
\begin{aligned}
\mathcal{P}\left[G_{u(1-\varepsilon)}^{\Sigma}(z) \leq G^{\hat{\zeta}}(z) \leq G_{u(1+\varepsilon)}^{\Sigma}(z) \text { for all } z \in \mathcal{K}\right] & \\
& \geq 1-c \exp \left(-\frac{c^{\prime}}{2} \varepsilon^{2} u s^{a}\right) .
\end{aligned}
$$

Proof: Observe that (4.1) implies

$$
\begin{aligned}
\int \mathcal{P}\left[G_{u(1-\varepsilon)}^{\Sigma}(z) \leq G^{\hat{\zeta}}(z) \leq G_{u(1+\varepsilon)}^{\Sigma}(z) \text { for all } z \in \mathcal{K}\right] \mathbb{P}_{V, \partial A_{2}}^{u}[\mathrm{~d} \hat{\zeta}] \\
\\
\geq 1-c \exp \left(-c^{\prime} \varepsilon^{2} u s^{a}\right) .
\end{aligned}
$$

Let

$$
\begin{aligned}
& \mathcal{A}:=\left\{\hat{\zeta} \in \mathcal{C}_{u}\left(V, \partial A_{2}\right)\right. \text { such that: } \\
& \left.\mathcal{P}\left[G_{u(1-\varepsilon)}^{\Sigma}(z) \leq G^{\hat{\zeta}}(z) \leq G_{u(1+\varepsilon)}^{\Sigma}(z) \text { for all } z \in \mathcal{K}\right] \geq 1-c \exp \left(-\frac{c^{\prime}}{2} \varepsilon^{2} u s^{a}\right)\right\} .
\end{aligned}
$$

Then (4.2) implies

$$
\mathbb{P}_{V, \partial A_{2}}^{u}[\mathcal{A}]+\left(1-c \exp \left(-\frac{c^{\prime}}{2} \varepsilon^{2} u s^{a}\right)\right)\left(1-\mathbb{P}_{V, \partial A_{2}}^{u}[\mathcal{A}]\right)
$$




$$
\geq 1-c \exp \left(-c^{\prime} \varepsilon^{2} u s^{a}\right)
$$

so that

$$
\mathbb{P}_{V, \partial A_{2}}^{u}[\mathcal{A}] \geq 1-\exp \left(-\frac{c^{\prime}}{2} \varepsilon^{2} u s^{a}\right) .
$$

This finishes the proof of the proposition.

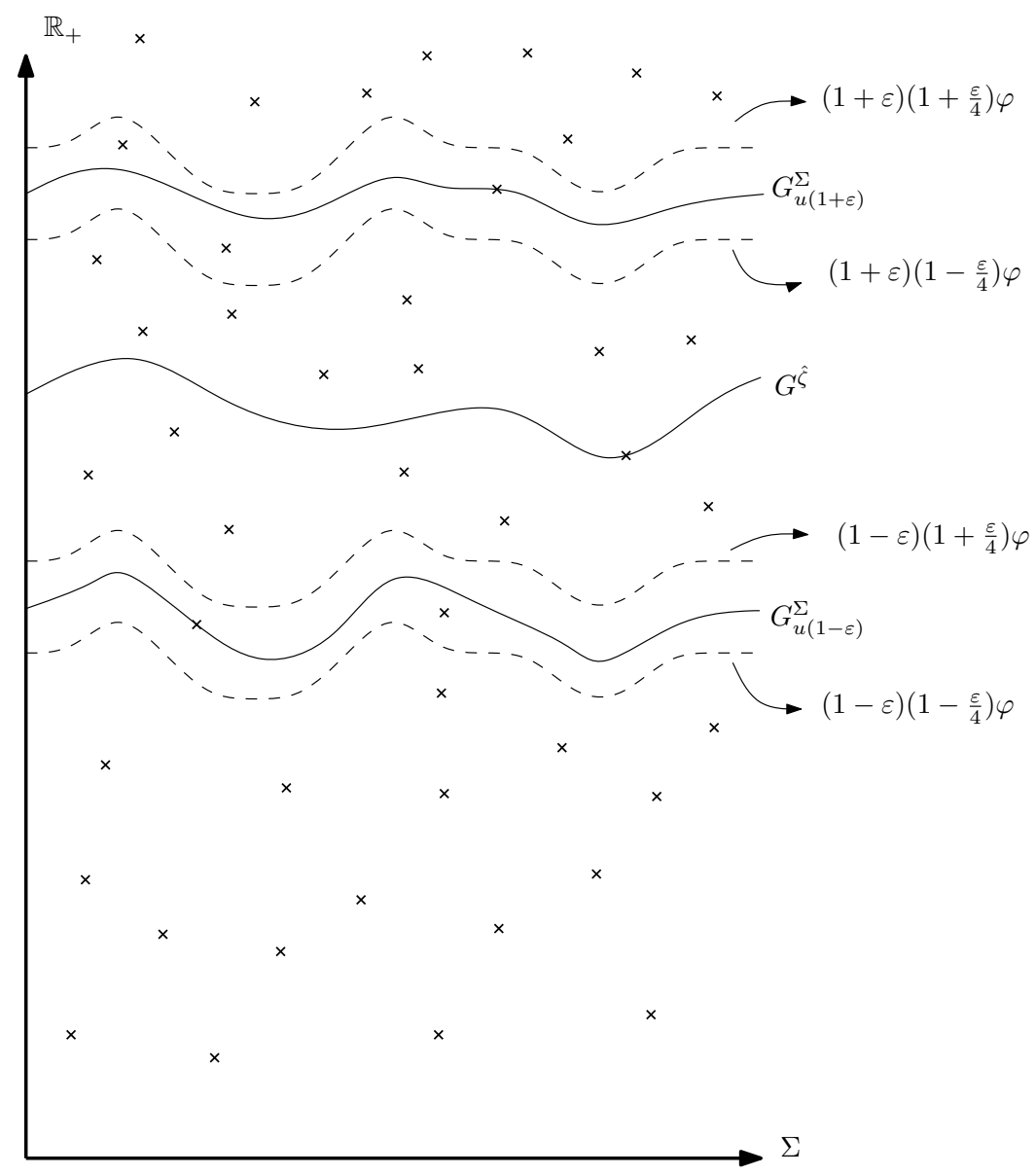

Figure 4.6. When the sequence $\hat{\zeta}$ belongs to a well behaved set $\mathcal{A}$, the decoupling probability is greater than $1-c \exp (-$ $\left.\frac{c^{\prime}}{2} \varepsilon^{2} u s^{a}\right)$. The symbol $\varphi$ in the figure stands for the function $u \operatorname{cap}(V) \pi(\Xi(z))$. The figure shows the decoupling event, where $G_{u(1-\varepsilon)}^{\Sigma}(z) \leq G^{\hat{\zeta}}(z) \leq G_{u(1+\varepsilon)}^{\Sigma}(z)$ for all $z \in \mathcal{K}$.

Proposition 4.2 implies that, for $\hat{\zeta} \in \mathcal{A}$, there exists a process $\left(\hat{\mathcal{I}}_{A_{1}}^{u}, u \geq 0\right)$ distributed as the random interlacements set intersected with $A_{1}$, and a coupling 
$\mathcal{P}$ such that, for all $\varepsilon>0$ sufficiently small and $r>0$ sufficiently big, we have

$$
\mathcal{P}\left[\hat{\mathcal{I}}_{A_{1}}^{u(1-\varepsilon)} \subseteq \mathcal{I}_{A_{1} \mid \hat{\zeta}}^{u} \subseteq \hat{\mathcal{I}}_{A_{1}}^{u(1+\varepsilon)}\right] \geq 1-c \exp \left(-\frac{c^{\prime}}{2} \varepsilon^{2} u s^{a}\right) .
$$

To complete the proof of our main theorem we need to show that a result similar to Proposition 4.2 remains valid under a different conditioning.

Let $A_{3} \subset A_{2}$ be such that $\left|\partial A_{3}\right|<\infty$. We enlarge our global probability space in order to make room for two new random elements. The first is $\Psi=\Psi\left(A_{3}\right)$, which is a random collection of points of $\partial A_{3}$ distributed as the intersection of $\partial A_{3}$ with the random walk trajectories that constitute the interlacements process $\mathcal{I}^{u}$. Given some possible realization $\hat{\psi}$ of $\Psi$, we define the second new random element to be $\mathcal{M}(\hat{\psi}) \in \mathcal{C}_{u}\left(V, \partial A_{2}\right)$, which is distributed as the clothesline process between $V$ and $\partial A_{2}$ generated by the interlacements trajectories at level $u$ conditioned on the fact that $\Psi=\hat{\psi}$. We can then construct a process $\mathcal{I}_{A_{1} \mid \hat{\psi}}^{u}$ which has the law of the the random interlacements process at level $u$ intersected with $A_{1}$ conditioned on the event where the intersections of the interlacements' random walk trajectories with $\partial A_{3}$ are given by $\hat{\psi}$. We obtain

$$
\begin{aligned}
\mathcal{P}\left[\hat{\mathcal{I}}_{A_{1}}^{u(1-\varepsilon)} \subseteq \mathcal{I}_{A_{1} \mid \hat{\psi}}^{u} \subseteq \hat{\mathcal{I}}_{A_{1}}^{u(1+\varepsilon)}\right] & =\sum_{\hat{\zeta} \in \mathcal{C}_{u}\left(V, \partial A_{2}\right)} \mathcal{P}\left[\hat{\mathcal{I}}_{A_{1}}^{u(1-\varepsilon)} \subseteq \mathcal{I}_{A_{1} \mid \hat{\psi}}^{u} \subseteq \hat{\mathcal{I}}_{A_{1}}^{u(1+\varepsilon)} \mid \mathcal{M}(\hat{\psi})=\hat{\zeta}\right] \\
& \times \mathcal{P}[\mathcal{M}(\hat{\psi})=\hat{\zeta}] \\
& =\sum_{\hat{\zeta} \in \mathcal{C}_{u}\left(V, \partial A_{2}\right)} \mathcal{P}\left[\hat{\mathcal{I}}_{A_{1}}^{u(1-\varepsilon)} \subseteq \mathcal{I}_{A_{1} \mid \hat{\zeta}}^{u} \subseteq \hat{\mathcal{I}}_{A_{1}}^{u(1+\varepsilon)}\right] \mathcal{P}[\mathcal{M}(\hat{\psi})=\hat{\zeta}] \\
& \geq\left(1-c \exp \left(-\frac{c^{\prime}}{2} \varepsilon^{2} u s^{a}\right)\right) \mathcal{P}[\mathcal{M}(\hat{\psi}) \in \mathcal{A}] .
\end{aligned}
$$

Let $\mathcal{E}$ be the set of all the $\hat{\psi}$ 's such that

$$
\mathcal{P}\left[\mathcal{M}(\hat{\psi}) \in \mathcal{A}^{C}\right] \geq \sqrt{\mathbb{P}_{V, \partial A_{2}}^{u}\left[\mathcal{A}^{C}\right]} .
$$

Since

$$
\mathbb{P}_{V, \partial A_{2}}^{u}\left[\mathcal{A}^{C}\right]=\int \mathcal{P}\left[\mathcal{M}(\hat{\psi}) \in \mathcal{A}^{C}\right] \mathcal{P}[\mathrm{d} \hat{\psi}] \geq \mathcal{P}[\mathcal{E}] \sqrt{\mathbb{P}_{V, \partial A_{2}}^{u}\left[\mathcal{A}^{C}\right]}
$$

we have

$$
\mathcal{P}[\mathcal{E}] \leq \sqrt{\mathbb{P}_{V, \partial A_{2}}^{u}\left[\mathcal{A}^{C}\right]} .
$$

We have proved the following theorem, which implies Theorem 1.1:

Theorem 4.3. Using the same notation as above, we have that, for constants $c, c^{\prime}>0$, there exists a set $\mathcal{E}$ made of possible values of $\Psi$ such that

$$
\mathcal{P}[\Psi \in \mathcal{E}] \geq 1-\exp \left(-\frac{c^{\prime}}{4} \varepsilon^{2} u s^{a}\right),
$$

and for all $\hat{\psi} \in \mathcal{E}$,

$$
\mathcal{P}\left[\hat{\mathcal{I}}_{A_{1}}^{u(1-\varepsilon)} \subseteq \mathcal{I}_{A_{1} \mid \hat{\psi}}^{u} \subseteq \hat{\mathcal{I}}_{A_{1}}^{u(1+\varepsilon)}\right] \geq 1-c \exp \left(-\frac{c^{\prime}}{2} \varepsilon^{2} u s^{a}\right) .
$$

We now present a brief proof of Theorem 1.2. 
Proof of Theorem 1.2: Note that, on equation (4.15), $\delta$ can be any real number greater than 0 , whereas in equation (4.16), we need to have $0<\delta<1$. Recall that $u^{\prime}>u>0$. We have, by substituting the appropriate $\delta$ in (4.17) and ignoring the union bound term $\mathrm{cr}^{2 d-2}$,

$$
\begin{aligned}
\mathcal{P}\left[G_{u}^{\Sigma}(z)<G_{u+u^{\prime}}^{\Sigma}(z)\right] \geq & 1-\mathcal{P}\left[G_{u}^{\Sigma}(z)>\left(u+u^{\prime} 4^{-1}\right) \operatorname{cap}(V) \pi(\Xi(z))\right] \\
& -\mathcal{P}\left[G_{u+u^{\prime}}^{\Sigma}(z)<2^{-1}\left(u+u^{\prime}\right) \operatorname{cap}(V) \pi(\Xi(z))\right] \\
\geq & 1-\exp \left(-\frac{c}{4}\left(u+u^{\prime}\right) s^{a}\right)-\exp \left(-\frac{c}{16} \frac{\left(u^{\prime}\right)^{2}}{u^{2}} u s^{a}\right) \\
\geq & 1-\exp \left(-c^{\prime} u^{\prime} s^{a}\right) .
\end{aligned}
$$

Now, proceeding in the same manner as we did in the proof of Theorem 4.3, we are able to prove Theorem 1.2.

Remark 4.4. As we previously stated, the above theorems are also valid for the unsmoothed versions of the sets $A_{1}^{\square}$ and $A_{2}^{\square}$. This result follows after employing the same argument present in the above proofs, where one considers the law of the entrance and exit point of the interlacements' trajectories in the smaller set (the smoothed version of $A_{2}^{\square}$ ) generating a law of entrance and exit points in the larger set (the unsmoothed version of $A_{2}^{\square}$ ), and then performing a computation similar to (4.4) in order to obtain good concentration inequalities for the "quenched" law. One then obtains in the same way the result for $A_{3} \subset A_{2}^{\square}$.

The remainder of the section is devoted to proving Proposition 4.1. We begin by stating the following bound

$$
\sup _{\substack{w^{\prime} \in V \\ y^{\prime} \in \partial A_{2}}} \hat{\mathbb{P}}_{w^{\prime}, y^{\prime}}\left[\Xi\left(w^{\prime}, y^{\prime}\right)=\left(w_{0}, y_{0}\right)\right] \leq c s^{-2(d-1)},
$$

for which the proof is technical and we thus postpone it to subsection $A .1$ of the appendix.

Let $z \in \Sigma$ be such that $\Xi(z)=\left(w_{0}, y_{0}\right) \in \partial A_{1} \times V$, and let $h:=\operatorname{dist}\left(w_{0}, y_{0}\right)$. We let $F\left(w_{0}, y_{0}\right)$ stand for $G(z)$, making explicit the dependence of the soft local time on the endvertices $\Xi(z)$. We define

$$
\pi\left(w_{0}, y_{0}\right):=\mathbb{E}\left(F\left(w_{0}, y_{0}\right)\right) .
$$

We define $f_{A_{1}}\left(w_{0}, y_{0}\right)$ to be the probability that the simple random walk started at $w_{0}$ visits $y_{0}$ before hitting $A_{2}$. We will prove in the appendix (see Section A.1, propositions A.1 and A.2) the following bounds for these probabilities:

(i) Given $\left(w_{0}, y_{0}\right) \in A_{1}^{\circ} \times V^{\circ}$, there are constants $c_{1}, c_{2}>0$ such that

$$
c_{1} \frac{s^{2}}{h^{d}} \leq f_{A_{1}^{\circ}}\left(w_{0}, y_{0}\right) \leq c_{2} \frac{s^{2}}{h^{d}} .
$$

(ii) Let $\left(w_{0}, y_{0}\right) \in A_{1}^{\square} \times V^{\square}$, and recall that $B_{\infty}(0, r+2 s)$ is the unsmoothed version of $A_{2}^{\square}$. Let $\mathfrak{H}_{i}^{d-1} ; i=1, \ldots, 2 d$; denote the $(d-1)$-dimensional hyperfaces of $B_{\infty}(0, r+2 s)$, and let $l_{i}^{w_{0}}:=\min \left\{\operatorname{dist}\left(w_{0}, \mathfrak{H}_{i}^{d-1}\right), h\right\}$, and $l_{i}^{y_{0}}:=\min \left\{\operatorname{dist}\left(y_{0}, \mathfrak{H}_{i}^{d-1}\right), h\right\}$. Then there are constants $c_{1}, c_{2}>0$ such that

$c_{1} \frac{l_{1}^{w_{0}} \ldots l_{2 d}^{w_{0}}}{h^{2 d}} \cdot \frac{1}{h^{d-2}} \cdot \frac{l_{1}^{y_{0}} \ldots l_{2 d}^{y_{0}}}{h^{2 d}} \leq f_{A_{1}^{\square}}\left(w_{0}, y_{0}\right) \leq c_{2} \frac{l_{1}^{w_{0}} \ldots l_{2 d}^{w_{0}}}{h^{2 d}} \cdot \frac{1}{h^{d-2}} \cdot \frac{l_{1}^{y_{0}} \ldots l_{2 d}^{y_{0}}}{h^{2 d}}$. 
The following lemma, whose proof we also postpone to the appendix (Section A.2), gives us an estimate on $\pi\left(w_{0}, y_{0}\right)$.

Lemma 4.5. Using the notation defined above we have, for constants $c_{1}, c_{2}, c_{3}, c_{4}>$ 0 :

(i) $c_{1} \operatorname{cap}(V)^{-1} s^{-1} f_{A_{1}}\left(w_{0}, y_{0}\right) \leq \pi\left(w_{0}, y_{0}\right) \leq c_{2} \operatorname{cap}(V)^{-1} s^{-1} f_{A_{1}}\left(w_{0}, y_{0}\right)$,

(ii) $\mathbb{E}\left(F\left(w_{0}, y_{0}\right)^{2}\right) \leq c_{3} \operatorname{cap}(V)^{-1} s^{-2 d+2} f_{A_{1}}\left(w_{0}, y_{0}\right)$.

Moreover, since $\operatorname{dist}\left(w_{0}, y_{0}\right) \geq s$, we have

(iii) $\sup _{w_{0}, y_{0}} \pi\left(w_{0}, y_{0}\right) \leq c_{4} \operatorname{cap}(V)^{-1} s^{-(d-1)}$.

We now provide a large deviation bound for $F\left(w_{0}, y_{0}\right)$.

Lemma 4.6. There are constants $c, c_{1}, c_{2}>0$ such that for every $\left(w_{0}, y_{0}\right) \in \partial A_{1} \times$ $V$, we have

$$
\mathcal{P}\left[F\left(w_{0}, y_{0}\right)>v c s^{-2(d-1)}\right] \leq c_{1} s^{2 d-3} f_{A_{1}}\left(w_{0}, y_{0}\right) \operatorname{cap}(V)^{-1} e^{-c_{2} v}
$$

for any $v \geq 2$ (we can also assume $c_{2} \leq 1$ without loss of generality).

Proof: In the proof of this particular result it will be important for us to distinguish between the constants. We will use Proposition 3.5 for $F\left(w_{0}, y_{0}\right)$, with

$$
\Gamma_{w_{0}, y_{0}}:=\left\{\left(w_{0}^{\prime}, y_{0}^{\prime}\right) \in \partial A_{1} \times V ; \max \left\{\left\|w_{0}^{\prime}-w_{0}\right\|,\left\|y_{0}^{\prime}-y_{0}\right\|\right\} \leq c_{4} s\right\},
$$

with $0<c_{4}<1$ defined in Section $A .3$ of the appendix.

Using the same notation as in Proposition 3.5, we note that (4.7) implies

$$
\ell \leq c s^{-2(d-1)}
$$

and observe that $\mu\left(\Gamma_{w_{0}, y_{0}}\right) \geq c_{5} s^{2(d-1)}$ for some constant $c_{5}>0$. Also, as can be seen in Section A.3 of the appendix, we have

$$
\alpha \geq c_{3}>0 .
$$

Chebyshev's inequality and Lemma 4.5 then imply

$$
\begin{aligned}
\mathcal{P}\left[T_{l}<\infty\right] \leq \mathcal{P}\left[F\left(w_{0}, y_{0}\right)>c s^{-2(d-1)}\right] & \leq \frac{\pi\left(w_{0}, y_{0}\right)}{c s^{-2(d-1)}} \\
& \leq c_{1} s^{2 d-3} f_{A_{1}}\left(w_{0}, y_{0}\right) \operatorname{cap}(V)^{-1} .
\end{aligned}
$$

We denote by $N\left(\Gamma_{w_{0}, y_{0}}\right)$ the number of times the simple random walk trajectory associated with $F\left(w_{0}, y_{0}\right)$ makes an excursion of the form $z^{\prime} \in \Sigma$ on $A_{2}^{C}$ such that $\Xi\left(z^{\prime}\right)=\left(w^{\prime}, y^{\prime}\right) \in \Gamma_{w_{0}, y_{0}}$. We also let $\eta_{w_{0}, y_{0}}$ stand for the number of points of the Poisson process associated with our soft local times that belong to $\Gamma_{w_{0}, y_{0}} \times\left[0, \frac{1}{2} v c c_{3} s^{-2(d-1)}\right]$. We note that both definitions are consistent with Proposition 3.5 and write

$$
\mathcal{P}\left[\eta_{w_{0}, y_{0}} \leq N\left(\Gamma_{w_{0}, y_{0}}\right)\right] \leq \mathcal{P}\left[\eta_{w_{0}, y_{0}} \leq \frac{c c_{3} c_{5} v}{4}\right]+\mathcal{P}\left[N\left(\Gamma_{w_{0}, y_{0}}\right) \geq \frac{c c_{3} c_{5} v}{4}\right] .
$$

We claim that both terms in the right side of the above inequality are exponentially small in $v$. To see why this is true, observe that:

- $\eta_{w_{0}, y_{0}}$ has Poisson distribution with parameter at least $\frac{c c_{3} c_{5} v}{2}$, and 
- every time the simple random walk associated with $F\left(w_{0}, y_{0}\right)$ hits $\partial A_{2}$, with uniform positive probability the walk never reaches $\Gamma_{w_{0}, y_{0}}$ again. This way $N\left(\Gamma_{w_{0}, y_{0}}\right)$ is dominated by a Geometric $\left(c_{6}\right)$ random variable, for some constant $c_{6}<1$.

Together with (4.12) and Proposition 3.5, this finishes the proof of the lemma.

We are now able to complete the proof of Proposition 4.1, that in turn implies our main results:

Proof of Proposition 4.1: Let $\Psi_{w_{0}, y_{0}}(\lambda)=\mathbb{E}\left(e^{\lambda F\left(w_{0}, y_{0}\right)}\right)$ be the moment generating function of $F\left(w_{0}, y_{0}\right)$. We are going to use the bounds above to estimate $\Psi_{w_{0}, y_{0}}$. It is elementary to obtain that $e^{t}-1 \leq t+t^{2}$ for $t \in[0,1]$. With this observation in mind, we write for $0 \leq \lambda \leq \frac{c_{2} s^{2(d-1)}}{2 c}$, where $c$ and $c_{2}$ are the same as in the theorem above:

$$
\begin{aligned}
& \Psi_{w_{0}, y_{0}}(\lambda)-1= \\
& =\mathbb{E}\left(e^{\lambda F\left(w_{0}, y_{0}\right)}-1\right) \mathbb{1}_{\lambda F\left(w_{0}, y_{0}\right) \leq 1}+\mathbb{E}\left(e^{\lambda F\left(w_{0}, y_{0}\right)}-1\right) \mathbb{1}_{\lambda F\left(w_{0}, y_{0}\right)>1} \\
& \leq \mathbb{E}\left(\lambda F\left(w_{0}, y_{0}\right)+\lambda^{2} F\left(w_{0}, y_{0}\right)^{2}\right)+\mathbb{E}\left(e^{\lambda F\left(w_{0}, y_{0}\right)}-1\right) \mathbb{1}_{\lambda F\left(w_{0}, y_{0}\right)>1} \\
& \leq \lambda \pi\left(w_{0}, y_{0}\right)+c_{1} \lambda^{2} \operatorname{cap}(V)^{-1} s^{-2 d+2} f_{A_{1}}\left(w_{0}, y_{0}\right)+\mathbb{E}\left(e^{\lambda F\left(w_{0}, y_{0}\right)}-1\right) \mathbb{1}_{\lambda F\left(w_{0}, y_{0}\right)>1} \\
& \leq \lambda \pi\left(w_{0}, y_{0}\right)+c^{\prime} \lambda^{2} \operatorname{cap}(V)^{-1} s^{-2 d+2} f_{A_{1}}\left(w_{0}, y_{0}\right)+\lambda \int_{\lambda^{-1}}^{\infty} e^{\lambda y} \mathcal{P}\left[F\left(w_{0}, y_{0}\right)>y\right] \mathrm{d} y \\
& \leq \lambda \pi\left(w_{0}, y_{0}\right) \\
& \quad+f_{A_{1}}\left(w_{0}, y_{0}\right) \operatorname{cap}(V)^{-1}\left(c^{\prime} \lambda^{2} s^{-2 d+2}+\lambda c^{\prime} s^{2 d-3} \int_{\lambda^{-1}}^{\infty} \exp \left(\frac{-c_{2} s^{2(d-1)} y}{2 c}\right) \mathrm{d} y\right) \\
& \leq \lambda \pi\left(w_{0}, y_{0}\right) \\
& \quad+f_{A_{1}}\left(w_{0}, y_{0}\right) \operatorname{cap}(V)^{-1}\left(c^{\prime} \lambda^{2} s^{-2 d+2}+c^{\prime} \lambda s^{-1} \exp \left(\frac{-c_{2} s^{2(d-1)} \lambda^{-1}}{2 c}\right)\right) \\
& \leq \lambda \pi\left(w_{0}, y_{0}\right)+c^{\prime} \lambda^{2} \operatorname{cap}(V)^{-1} s^{-2 d+2} f_{A_{1}}\left(w_{0}, y_{0}\right),
\end{aligned}
$$

where we used Lemma 4.5 and Lemma 4.6. Now since $e^{-t}-1 \leq-t+t^{2}$ for all $t \geq 0$, we obtain for $\lambda \geq 0$

$$
\Psi_{w_{0}, y_{0}}(-\lambda)-1 \leq-\lambda \pi\left(w_{0}, y_{0}\right)+c \lambda^{2} \operatorname{cap}(V)^{-1} s^{-2 d+2} f_{A_{1}}\left(w_{0}, y_{0}\right)
$$

(the large deviation bound of Lemma 4.6 is not necessary is this case).

Observe that if $\left(\chi_{j}, j \geq 1\right)$ are i.i.d. random variables with common moment generating function $\Psi$ and $N$ is an independent Poisson random variable with parameter $\theta$, then

$$
\mathbb{E} \exp \left(\lambda \sum_{j=1}^{N} \chi_{j}\right)=e^{(\theta(\Psi(\lambda)-1))}
$$

We let $F_{j}\left(w_{0}, y_{0}\right)$ denote the expectation $\mathbb{E}\left(G^{j}(z)\right)$ defined in (3.16), when $z \in \Sigma$ is such that $\Xi(z)=\left(w_{0}, y_{0}\right)$. Using Lemma 4.5 and (4.13), we have, for $N_{\hat{u}}^{V} \stackrel{d}{=}$ 
$\operatorname{Poisson}(\hat{u} \operatorname{cap}(V))$ and any $\delta>0$

$$
\begin{aligned}
\mathcal{P}\left[G_{\hat{u}}^{\Sigma}(z) \geq(\right. & \left.+\delta) \hat{u} \operatorname{cap}(V) \pi\left(w_{0}, y_{0}\right)\right]= \\
& =\mathcal{P}\left[\sum_{j=1}^{N_{\hat{u}}^{V}} F_{j}\left(w_{0}, y_{0}\right) \geq(1+\delta) \hat{u} \operatorname{cap}(V) \pi\left(w_{0}, y_{0}\right)\right] \\
& \leq \frac{\mathbb{E}\left(\exp \left(\lambda \sum_{j=1}^{N_{\hat{u}}^{V}} F_{j}\left(w_{0}, y_{0}\right)\right)\right)}{\exp \left(\lambda(1+\delta) \hat{u} \operatorname{cap}(V) \pi\left(w_{0}, y_{0}\right)\right)} \\
& \leq \exp \left(-\lambda(1+\delta) \hat{u} \operatorname{cap}(V) \pi\left(w_{0}, y_{0}\right)+\hat{u} \operatorname{cap}(V)\left(\Psi_{w_{0}, y_{0}}(\lambda)-1\right)\right) \\
& \leq \exp \left(-\left(\lambda \delta \hat{u} \operatorname{cap}(V) \pi\left(w_{0}, y_{0}\right)-c^{\prime} \lambda^{2} \hat{u} s^{-2 d+2} f_{A_{1}}\left(w_{0}, y_{0}\right)\right)\right) \\
& \leq \exp \left(-\left(\lambda \delta \hat{u} c s^{-1} f_{A_{1}}\left(w_{0}, y_{0}\right)-c^{\prime} \lambda^{2} \hat{u} s^{-2 d+2} f_{A_{1}}\left(w_{0}, y_{0}\right)\right)\right)
\end{aligned}
$$

Analogously, with (4.14) instead of (4.13), we obtain

$$
\begin{aligned}
\mathcal{P}\left[G_{\hat{u}}^{\Sigma}(z)\right. & \left.\leq(1-\delta) \hat{u} \operatorname{cap}(V) \pi\left(w_{0}, y_{0}\right)\right] \\
& \leq \exp \left(-\left(\lambda \delta \hat{u} c s^{-1}-c^{\prime} \lambda^{2} \hat{u} s^{-2 d+2}\right) f_{A_{1}}\left(w_{0}, y_{0}\right)\right) .
\end{aligned}
$$

We choose $\lambda=c_{7} \delta s^{2 d-3}$ with $c_{7}$ small enough so that $\lambda \leq \frac{c_{2} s^{2(d-1)}}{2 c}$, and observe that the bounds for $f_{A_{1}}\left(w_{0}, y_{0}\right)$ given in (4.9) and (4.10) imply

$$
\begin{aligned}
& \inf _{w_{0}, y_{0}} f_{A_{1}^{\square}}\left(w_{0}, y_{0}\right) \geq c s^{2 d} r^{-3 d+2}, \\
& \inf _{w_{0}, y_{0}} f_{A_{1}^{\circ}}\left(w_{0}, y_{0}\right) \geq c s^{2} r^{-d} .
\end{aligned}
$$

Recall the definition of $b_{\odot}$, a number such that

$$
1 \leq b_{\odot}<\frac{2 d-2}{d}
$$

and the definition of $b_{\square}$, a number such that

$$
1 \leq b_{\square}<\frac{4 d-4}{3 d-2} .
$$

Recall that $s=s(r) \asymp r^{\frac{1}{b}}$. Then, since $a_{\circ}:=2 d-2-d b_{\bigcirc}>0$ and $a_{\square}:=$ $4 d-4-3 d b_{\square}+2 b_{\square}>0$, we have

$$
\mathcal{P}\left[G_{\hat{u}}^{\Sigma}(z) \geq(1+\delta) \hat{u} \operatorname{cap}(V) \pi\left(w_{0}, y_{0}\right)\right] \leq \exp \left(-c \delta^{2} \hat{u} s^{a}\right) .
$$

Using the union bound (note that $\partial A_{1} \times V$ has $O\left(r^{2(d-1)}\right)$ elements),

$$
\begin{aligned}
& \mathcal{P}\left[(1-\delta) \hat{u} \operatorname{cap}(V) \pi(\Xi(z)) \leq G_{\hat{u}}^{\Sigma}(z) \leq(1+\delta) \hat{u} \operatorname{cap}(V) \pi(\Xi(z)), \text { for all } z \in \mathcal{K}\right] \\
& \geq 1-c r^{2(d-1)} \exp \left(-c^{\prime} \delta^{2} \hat{u} s^{a}\right) .
\end{aligned}
$$

Observe that we can suppose $c^{\prime} \leq 1$ without loss of generality. Using (4.17) and the union bound we obtain, for $\varepsilon \in(0,1 / 4)$,

$$
\mathcal{P}\left[D_{u}^{\varepsilon / 4}, D_{u(1-\varepsilon)}^{\varepsilon / 4}, D_{u(1+\varepsilon)}^{\varepsilon / 4}\right] \geq 1-c r^{2(d-1)} \exp \left(-c^{\prime} \varepsilon^{2} u s^{a}\right) .
$$

Since $s=s(r) \asymp r^{\frac{1}{b}}$, by replacing the constants $c$ and $c^{\prime}$ in the above equation we obtain (4.1) and finish the proof of Proposition 4.1. 


\section{Appendix A. Technical estimates}

A.1. Bounding the relevant probabilities. For $w_{0} \in \partial A_{1}$ and $y_{0} \in V$ we want to study the supremum

$$
\sup _{\substack{w \in V \\ w^{\prime} \in V \\ y^{\prime} \in \partial A_{2}}} \hat{\mathbb{P}}_{w^{\prime}, y^{\prime}}\left[\Xi\left(w^{\prime}, y^{\prime}\right)=\left(w_{0}, y_{0}\right)\right]
$$

Furthermore, we need to bound the "hanging" probability $\hat{\mathbb{P}}_{w, y}\left[\Xi(w, y)=\left(w_{0}, y_{0}\right)\right]$ for arbitrary $w \in V$ and $y \in \partial A_{2}$ from above and from bellow.

Given a finite nearest neighbor path $\gamma$, we denote by $|\gamma|$ its length. We will say that a path $\gamma$ belongs to an event $E$ if $E$ occurs every time the simple random walk $\left(X_{k}, k \geq 0\right)$ first $|\gamma|$ steps coincide with $\gamma$. We also let $\mathbb{P}_{x}[\gamma]$ denote the probability that the first $|\gamma|$ steps of the simple random walk started at $x$ coincide with $\gamma$.

In order to avoid a cumbersome notation we now introduce what, hopefully, will be a simpler way to denote our events of interest. For $w, y_{0} \in V, w_{0} \in \partial A_{1}$ and $y \in \partial A_{2}$ we define:

- $w \stackrel{1}{\rightarrow} w_{0}$ : The collection of all finite nearest-neighbor trajectories starting at $w$ that do not reach neither $\partial A_{1}$ nor $\partial A_{2}$, except at its ending point $w_{0} \in \partial A_{1}$. Note that this collection can be thought of as the event where the simple random walk started at $w$ hits $\partial A_{1}$ for the first time at $w_{0}$ before reaching $\partial A_{2}$.

- $w_{0} \stackrel{2}{\rightarrow} y_{0}$ : The collection of all finite nearest-neighbor trajectories starting at $w_{0}$ and ending at $y_{0}$ without reaching $\partial A_{2}$.

- $y_{0} \stackrel{3}{\rightarrow} y$ : The collection of all finite nearest-neighbor trajectories starting at $y_{0}$ that hit $\partial A_{2}$ for the first time at $y$ before returning to $V$. Note that this collection can be thought of as the event where the simple random walk started at $y_{0}$ hits $\partial A_{2}$ before returning to $V$ and its entrance point in $\partial A_{2}$ is $y$.

- $w \stackrel{4}{\rightarrow} y$ : The event where the entrance point in $\partial A_{2}$ of the simple random walk started at $w$ is $y$. This event can also be regarded as a collection of simple random walk trajectories starting at $w$ and hitting $\partial A_{2}$ for the first time at $y$.

We also let $w \stackrel{1}{\rightarrow} w_{0} \stackrel{2}{\rightarrow} y_{0} \stackrel{3}{\rightarrow} y$ be the "concatenation" of the first three collections, where the first trajectory's ending point becomes the second trajectory's starting point and so on. That is, if $\gamma \in w \stackrel{1}{\rightarrow} w_{0} \stackrel{2}{\rightarrow} y_{0} \stackrel{3}{\rightarrow} y$ then $\gamma$ is the concatenation of three distinct paths: $\gamma_{1} \in w \stackrel{1}{\rightarrow} w_{0}, \gamma_{2} \in w_{0} \stackrel{2}{\rightarrow} y_{0}, \gamma_{3} \in y_{0} \stackrel{3}{\rightarrow} y$. With our new notation the hanging probability becomes

$$
\begin{aligned}
\hat{\mathbb{P}}_{w, y}\left[\Xi(w, y)=\left(w_{0}, y_{0}\right)\right] & =\mathbb{P}_{w}\left[w \stackrel{1}{\rightarrow} w_{0} \stackrel{2}{\rightarrow} y_{0} \stackrel{3}{\rightarrow} y \mid w \stackrel{4}{\rightarrow} y\right] \\
& =\frac{\mathbb{P}_{w}\left[w \stackrel{1}{\rightarrow} w_{0} \stackrel{2}{\rightarrow} y_{0} \stackrel{3}{\rightarrow} y\right]}{\mathbb{P}_{w}[w \stackrel{4}{\rightarrow} y]}
\end{aligned}
$$




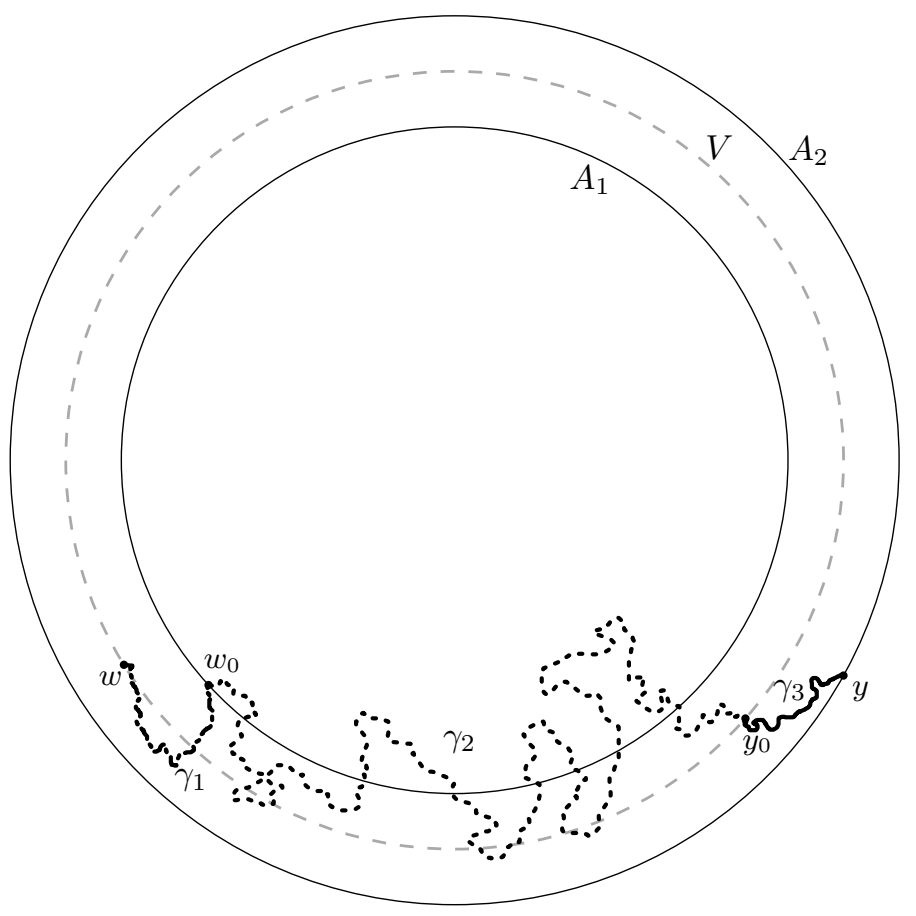

FIgURE A.7. $\gamma$ as the concatenation of the three paths $\gamma_{1}, \gamma_{2}$ and $\gamma_{3}$.

We have

$$
\begin{aligned}
\mathbb{P}_{w}\left[w \stackrel{1}{\rightarrow} w_{0} \stackrel{2}{\rightarrow} y_{0} \stackrel{3}{\rightarrow} y\right] & =\sum_{\gamma \in w \stackrel{1}{\rightarrow} w_{0} \stackrel{2}{\rightarrow} y_{0} \stackrel{3}{\rightarrow} y} \frac{1}{(2 d)^{|\gamma|}} \\
& =\sum_{\gamma_{1} \in w^{1} \rightarrow w_{0}} \frac{1}{(2 d)^{\left|\gamma_{1}\right|}} \sum_{\gamma_{2} \in w_{0} \stackrel{2}{\rightarrow} y_{0}} \frac{1}{(2 d)^{\left|\gamma_{2}\right|}} \sum_{\gamma_{3} \in y_{0} \stackrel{3}{\rightarrow} y} \frac{1}{(2 d)^{\left|\gamma_{3}\right|}} .
\end{aligned}
$$

Let us focus on the second sum, $\sum_{\gamma_{2} \in w_{0}} \stackrel{2}{\rightarrow} y_{0} \frac{1}{(2 d)^{\left|\gamma_{2}\right|}}$, for a moment. Each $\gamma_{2} \in$ $w_{0} \stackrel{2}{\rightarrow} y_{0}$ can be seen as the concatenation of one path $\gamma_{2}^{0}$ responsible for the walk's first visit to $y_{0}$ and a sequence of paths $\gamma_{2}^{1}, \ldots, \gamma_{2}^{k}$ associated with the returns the walk makes to $y_{0}$ before hitting $\partial A_{2}$, see Figure A.8. So

$$
\sum_{\gamma_{2}} \mathbb{P}_{w_{0}}\left[\gamma_{2}\right]=\sum_{\gamma_{2}^{0}} \mathbb{P}_{w_{0}}\left[\gamma_{2}^{0}\right] \sum_{k \geq 1} \sum_{\gamma_{2}^{1}, \ldots, \gamma_{2}^{k}} \mathbb{P}_{y_{0}}\left[\gamma_{2}^{1}\right] \ldots \mathbb{P}_{y_{0}}\left[\gamma_{2}^{k}\right]
$$

But for a fixed $k_{0}>0$, the last sum $\sum_{k \geq k_{0}} \sum_{\gamma_{2}^{1}, \ldots, \gamma_{2}^{k_{0}}} \mathbb{P}_{y_{0}}\left[\gamma_{2}^{1}\right] \ldots \mathbb{P}_{y_{0}}\left[\gamma_{2}^{k_{0}}\right]$ equals the probability that the simple random walk started at $y_{0}$ returns to $y_{0}$ at least $k_{0}$ times before hitting $\partial A_{2}$. Since the walk is transient, we can use the strong Markov property to show that there exists a constant $0<c<1$ such that

$$
\sum_{k \geq k_{0}} \sum_{\gamma_{2}^{1}, \ldots, \gamma_{2}^{k_{0}}} \mathbb{P}_{y_{0}}\left[\gamma_{2}^{1}\right] \ldots \mathbb{P}_{y_{0}}\left[\gamma_{2}^{k_{0}}\right]<c^{k_{0}}
$$




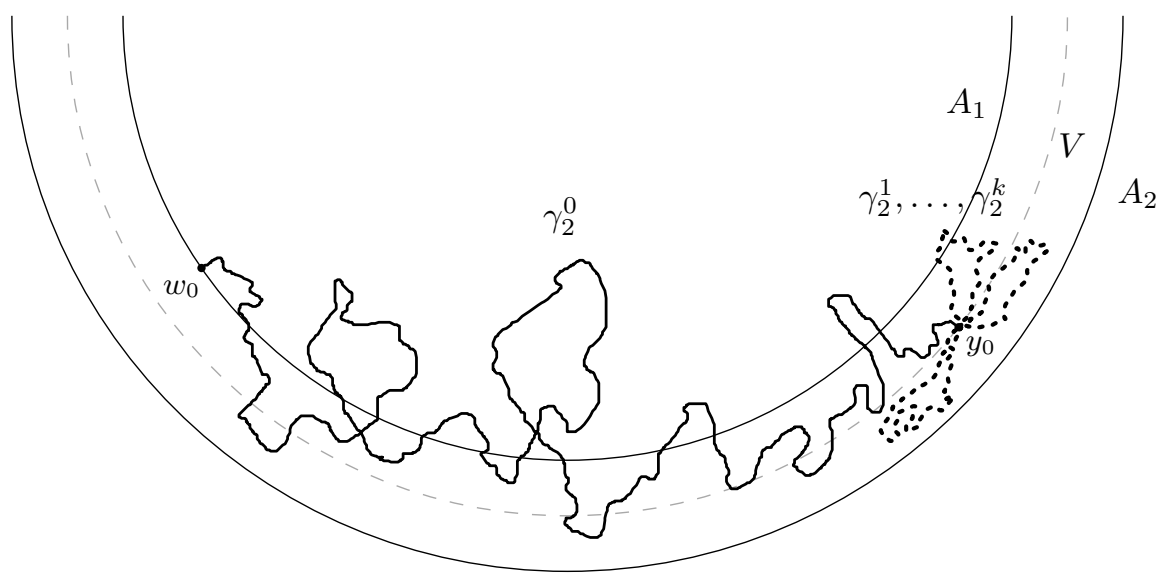

FiguRE A.8. $\gamma_{2}$ as the concatenation of the paths $\gamma_{2}^{0}, \gamma_{2}^{1}, \ldots, \gamma_{2}^{k}$.

We have thus shown the existence of a constant $c>0$ such that

$$
\sum_{\gamma_{2}^{0}} \mathbb{P}_{w_{0}}\left[\gamma_{2}^{0}\right] \leq \sum_{\gamma_{2}} \mathbb{P}_{w_{0}}\left[\gamma_{2}\right] \leq c \sum_{\gamma_{2}^{0}} \mathbb{P}_{w_{0}}\left[\gamma_{2}^{0}\right]
$$

where $\gamma_{2}^{0}$ represents any nearest neighbor path that starts at $w_{0}$ and ends at its only visit to $y_{0}$, without ever reaching $\partial A_{2}$. Let us update our collection's definition in view of this last computation. We denote by

- $w_{0} \stackrel{2^{\prime}}{\rightarrow} y_{0}$ : The collection of all finite nearest-neighbor paths starting at $w_{0}$ and ending at their first visit to $y_{0}$, without hitting $\partial A_{2}$. This collection now can be thought of as the event where the simple random walk started at $w_{0}$ makes a visit to $y_{0}$ before hitting $\partial A_{2}$.

Combining (A.3) with $(A .6)$ we get

$$
\mathbb{P}_{w}\left[w \stackrel{1}{\rightarrow} w_{0} \stackrel{2}{\rightarrow} y_{0} \stackrel{3}{\rightarrow} y\right] \leq c \mathbb{P}_{w}\left[w \stackrel{1}{\rightarrow} w_{0}\right] \mathbb{P}_{w_{0}}\left[w_{0} \stackrel{2^{\prime}}{\rightarrow} y_{0}\right] \mathbb{P}_{y_{0}}\left[y_{0} \stackrel{3}{\rightarrow} y\right] .
$$

Our work will now reside in giving upper bounds for these probabilities.

Using standard techniques from simple random walk theory, such as exit and entrance measure estimates, the Green function's estimate (2.1) and Lemma 2.1, one can prove the following result:

Proposition A.1. Recall that $h:=\operatorname{dist}\left(w_{0}, y_{0}\right)$ and define

$$
\begin{aligned}
& h_{1}:=\operatorname{dist}\left(w, w_{0}\right) \\
& h_{3}:=\operatorname{dist}\left(y, y_{0}\right) \\
& h_{4}:=\operatorname{dist}(w, y)
\end{aligned}
$$

Regarding the sets $A_{1}^{\circ}, V^{\circ}$ and $A_{2}^{\circ}$, we have that there exist constants $c_{k}>0$, $k=1, \ldots, 9$, such that

$$
\begin{array}{r}
c_{1} e^{\frac{-c_{2} h_{1}}{s}} s^{-(d-1)} \leq \mathbb{P}_{w}\left[w \stackrel{1}{\rightarrow} w_{0}\right] \leq c_{2} e^{\frac{-c_{2} h_{1}}{s}} s^{-(d-1)}, \\
c_{3} e^{\frac{-c_{4} h_{3}}{s}} s^{-(d-1)} s^{-1} \leq \mathbb{P}_{w}\left[y \stackrel{3}{\rightarrow} y_{0}\right] \leq c_{4} e^{\frac{-c_{4} h_{3}}{s}} s^{-(d-1)} s^{-1},
\end{array}
$$




$$
\begin{gathered}
c_{5} \frac{s^{2}}{h^{d}} \leq \mathbb{P}_{w_{0}}\left[w_{0} \stackrel{2^{\prime}}{\rightarrow} y_{0}\right] \leq c_{6} \frac{s^{2}}{h^{d}} \\
c_{7} \frac{s}{h_{4}^{d}} \leq \mathbb{P}_{w_{0}}[w \stackrel{4}{\rightarrow} y] \leq c_{8} \frac{s}{h_{4}^{d}} . \\
\sup _{\substack{w \in V \\
y \in \partial A_{2}}} \mathbb{P}_{w}\left[w \stackrel{1}{\rightarrow} w_{0} \stackrel{2}{\rightarrow} y_{0} \stackrel{3}{\rightarrow} y \mid w \stackrel{4}{\rightarrow} y\right] \leq c_{9} s^{-2(d-1)} .
\end{gathered}
$$

We will briefly describe how the random walk trajectories should behave in order to satisfy the conditions in each of the above events, which should give a general idea about how to prove the above result rigorously. We will omit the details for the sake of brevity.

Sketch of the proof: In (A.9) the walk started at $w$ has to cross $\sim h_{1} s^{-1}$ subsets of $A_{2}^{C} \backslash A_{1}$ with diameter $\sim s$ without reaching either $\partial A_{2}$ or $\partial A_{1}$, which implies the exponential factor; and then after coming within distance $\sim s$ of $w_{0}$ it has to enter $\partial A_{1}$ though $w_{0}$, from which the $\sim s^{-(d-1)}$ term comes from.

The bound (A.10) follows from similar reasoning as in (A.9), but now the walk started at $y_{0}$ pays a price $\sim s^{-1}$ in order to reach $y$ without returning to $V$.

In (A.11), the walk started at $w_{0}$ has to escape the proximity with $\partial A_{2}$, which it does with probability $\sim s h^{-1}$, then reach a ball with radius $\sim s$ centered at $y_{0}$ without ever reaching $\partial A_{2}$, which costs $\sim s^{d-1} h^{-(d-1)}$, before finally reaching $y_{0}$ from this ball, which has a cost of $s^{-(d-2)}$.

In (A.12), the random walk started at $w$ escapes the proximity with $\partial A_{2}$ with probability $\sim s h_{4}^{-1}$ and then hits $\partial A_{2}$ for the first time at $y$ with probability $h_{4}^{-(d-1)}$.

Finally, (A.13) is implied by the above bounds in an elementary way.

We will now focus on sets $A_{1}^{\square}, V^{\square}$ and $A_{2}^{\square}$, and the related simple random walk probabilities. We will continue to use the notation introduced in (A.8) in this new context.

$\boldsymbol{w} \stackrel{\mathbf{1}}{\rightarrow} \boldsymbol{w}_{\mathbf{0}}, \boldsymbol{y}_{\mathbf{0}} \stackrel{\mathbf{3}}{\rightarrow} \boldsymbol{y}$ : The bounds for the probabilities of these events have the same order as the bounds for the corresponding events when the underlying sets are Euclidean balls, and their proofs are essentially the same.

$\boldsymbol{w}_{\mathbf{0}} \stackrel{\boldsymbol{2}^{\prime}}{\rightarrow} \boldsymbol{y}_{\mathbf{0}}$ : If $h<100 \mathrm{~s}$, an application of a Green's function estimate gives the desired result. We then assume $h>100 s$. Define $\tilde{B}_{x}$ to be the discrete ball in the $\ell_{\infty}$-norm centered in $x$ with radius $\frac{h}{4 \sqrt{d}}$.

We will break up the path $\gamma_{2}^{0} \in w_{0} \stackrel{2^{\prime}}{\rightarrow} y_{0}$ into pieces that are easier to work with. Let $w_{4} \in \partial \tilde{B}_{w_{0}} \cap A_{2}^{C}, y_{4} \in \partial \tilde{B}_{y_{0}} \cap A_{2}^{C}$. We define the collection of finite paths:

- $w_{0} \stackrel{5}{\rightarrow} w_{4}$ : The collection of all finite nearest-neighbor paths starting at $w_{0}$ whose only intersection with $\partial \tilde{B}_{w_{0}} \cup \partial A_{2}$ is at its ending point $w_{4} \in$ $\partial \tilde{B}_{w_{0}} \cap A_{2}^{C}$.

- $w_{4} \stackrel{6}{\rightarrow} y_{4}$ : The collection of finite nearest-neighbor paths starting at $w_{4}$ and ending at $y_{4}$, without intersecting $\partial A_{2}$.

- $y_{4} \stackrel{7}{\rightarrow} y_{0}$ : The collection of all finite nearest-neighbor paths that start at $y_{4}$, never return to $\partial \tilde{B}_{y_{0}} \cap A_{2}^{C}$, and end at $y_{0}$ without ever reaching $\partial A_{2}$. 


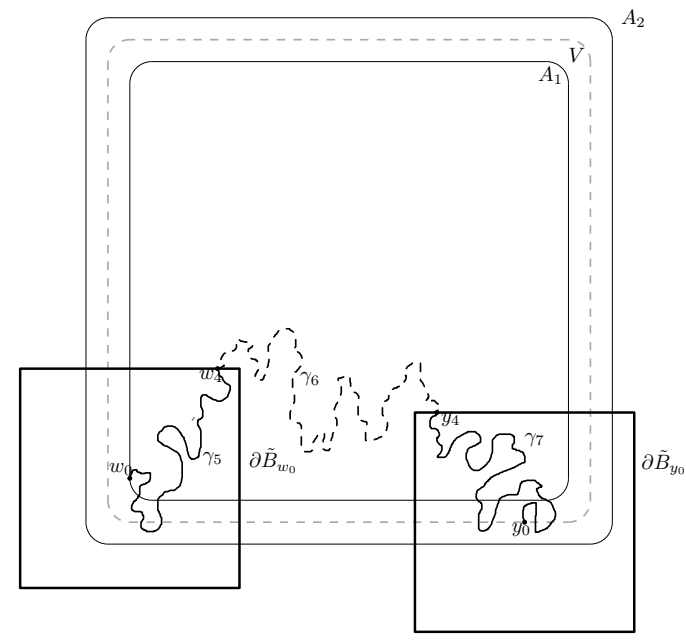

Figure A.9. Definition of the paths $\gamma_{5}, \gamma_{6}$ and $\gamma_{7}$.

As before, we denote by $w_{0} \stackrel{5}{\rightarrow} w_{4} \stackrel{6}{\rightarrow} y_{4} \stackrel{7}{\rightarrow} y_{0}$ the concatenation of these three collections.

Analogously to what we noted at the start of this section, we observe that $\gamma_{2}^{0} \in$ $w_{0} \stackrel{2^{\prime}}{\rightarrow} y_{0}$ if and only if there exists $w_{4} \in \partial \tilde{B}_{w_{0}} \cap A_{2}^{C}$ and $y_{4} \in \partial \tilde{B}_{y_{0}} \cap A_{2}^{C}$ such that $\gamma_{2}^{0}$ is the concatenation of three paths: $\gamma_{5} \in w_{0} \stackrel{5}{\rightarrow} w_{4}, \gamma_{6} \in w_{4} \stackrel{6}{\rightarrow} y_{4}$, and $\gamma_{7} \in y_{4} \stackrel{7}{\rightarrow} y_{0}$.

We also define

- $w_{4} \stackrel{6^{\prime}}{\rightarrow} y_{4}$ The collection of finite simple random walk trajectories starting at $w_{4}$ and ending at its first visit to $y_{4}$ without intersecting $\partial A_{2}$. This collection can also be seen as the event where the simple random walk started at $w_{4}$ visits $y_{4}$ before it hits $\partial A_{2}$.

Using the same argument used to obtain the bound (A.6), we can find a constant $c>1$ such that

$$
\mathbb{P}_{w_{4}}\left[w_{4} \stackrel{6}{\rightarrow} y_{4}\right] \leq \sum_{\gamma_{6} \in w_{4} \stackrel{6}{\rightarrow} y_{4}} \frac{1}{(2 d)^{\left|\gamma_{6}\right|}} \leq c \mathbb{P}_{w_{4}}\left[w_{4} \stackrel{6^{\prime}}{\rightarrow} y_{4}\right] .
$$

We then have

$$
\begin{aligned}
\mathbb{P}_{w_{0}}\left[w_{0} \stackrel{2^{\prime}}{\rightarrow} y_{0}\right] & =\sum_{\substack{w_{4} \in \partial \tilde{B}_{w_{0}} \cap A_{2}^{C} \\
\gamma_{5} \in w_{0} \stackrel{5}{\rightarrow} w_{4}}} \frac{1}{(2 d)^{\left|\gamma_{5}\right|}} \sum_{\substack{y_{4} \in \partial \tilde{B}_{y_{0}} \cap A_{2}^{C} \\
\gamma_{6} \in w_{4} \stackrel{6}{\rightarrow} y_{4}}} \frac{1}{(2 d)^{\left|\gamma_{6}\right|}} \sum_{\gamma_{7} \in y_{4} \stackrel{6}{\rightarrow} y_{0}} \frac{1}{(2 d)^{\left|\gamma_{7}\right|}} \\
& \leq c \sum_{w_{4}} \mathbb{P}_{w_{0}}\left[w_{0} \stackrel{5}{\rightarrow} w_{4}\right] \sum_{y_{4}} \mathbb{P}_{w_{4}}\left[w_{4} \stackrel{6^{\prime}}{\rightarrow} y_{4}\right] \mathbb{P}_{y_{4}}\left[y_{4} \stackrel{7}{\rightarrow} y_{0}\right] .
\end{aligned}
$$

We then use the Green's function estimate (2.1) to bound $\mathbb{P}_{w_{4}}\left[w_{4} \stackrel{6^{\prime}}{\rightarrow} y_{4}\right]$ by $c h^{d-2}$ (note that $\left.\operatorname{dist}\left(w_{4}, y_{4}\right)=O(h)\right)$. Using this bound on the above inequality, we 
obtain

$$
\mathbb{P}_{w_{0}}\left[w_{0} \stackrel{2^{\prime}}{\rightarrow} y_{0}\right] \leq c h^{d-2} \sum_{w_{4}} \mathbb{P}_{w_{0}}\left[w_{0} \stackrel{5}{\rightarrow} w_{4}\right] \sum_{y_{4}} \mathbb{P}_{y_{4}}\left[y_{4} \stackrel{7}{\rightarrow} y_{0}\right]
$$

Note that

$$
\sum_{w_{4}} \mathbb{P}_{w_{0}}\left[w_{0} \stackrel{5}{\rightarrow} w_{4}\right]=\mathbb{P}_{w_{0}}\left[H_{\partial \tilde{B}_{w_{0}}} \leq H_{\partial A_{2}}\right],
$$

and using the simple random walk's reversibility, we also have

$$
\sum_{y_{4}} \mathbb{P}_{y_{4}}\left[y_{4} \stackrel{5}{\rightarrow} y_{0}\right]=\mathbb{P}_{y_{0}}\left[H_{\partial \tilde{B}_{y_{0}}} \leq H_{\partial A_{2}}\right]
$$

so that we obtain the following bound

$$
\mathbb{P}_{w_{0}}\left[w_{0} \stackrel{2^{\prime}}{\rightarrow} y_{0}\right] \leq \frac{c}{h^{d-2}} \mathbb{P}_{w_{0}}\left[H_{\partial \tilde{B}_{w_{0}}} \leq H_{\partial A_{2}}\right] \mathbb{P}_{y_{0}}\left[H_{\partial \tilde{B}_{y_{0}}} \leq H_{\partial A_{2}}\right]
$$

We still have to obtain bounds for these last two probabilities. Since they are similarly defined, the bound for both of them follows from the same arguments, and thus we will only provide a bound for $\mathbb{P}_{w_{0}}\left[H_{\partial \tilde{B}_{w_{0}}} \leq H_{\partial A_{2}}\right]$.

We will do so by looking at the projections of the random walk trajectory in each of the $d$ orthogonal axes. Since we will need to look at these projections independently, we will change our object of study from the simple random walk on $\mathbb{Z}^{d}$ to the continuous time simple random walk on $\mathbb{Z}^{d}$ with waiting times between steps distributed as $\operatorname{Exp}(1)$ random variables. Since we will be studying properties of the random walk's trajectories, this change of framework will in no way impact the probabilities of interest.

We recall that $B_{\infty}(0, r+2 s)$ is the unsmoothed version of $A_{2}^{C}$. To simplify our notation we will work with the following translate of $B_{\infty}(0, r+2 s)$ :

$$
\mathfrak{H}_{r+2 s}:=\left\{\left(x_{1}, \ldots, x_{d}\right) \in \mathbb{Z}^{d}: 0 \leq x_{i} \leq 2 r+4 s+1, \text { for all } i=1, \ldots, d\right\} .
$$

Without loss of generality we assume that $0 \in \mathbb{Z}^{d}$ is the point belonging to

$$
\{0,2 r+4 s+1\}^{d}
$$

which is closest to $w_{0}$. We denote $w_{0} \equiv\left(w_{0}^{1}, \ldots, w_{0}^{d}\right)$. The projection of the simple random walk in the $j$ th coordinate axis is itself a continuous time random walk started at $w_{0}^{j}$ with waiting time between jumps given by a $\operatorname{Exp}(d)$ random variable, and, as we already noted, these projected random walks are independent from each other.

Given $w_{0}$, we divide the $d$ directions of $\mathbb{Z}^{d}$ in two kinds. The first kind will be such that $w_{0}^{j} \leq h(4 \sqrt{d})^{-1}$, the second will be such that $w_{0}^{j}>h(4 \sqrt{d})^{-1}$. We assume without loss of generality the first $d_{0}$ directions to be of the first kind and the remaining directions to be of the second kind. Our goal now is establishing the following bound:

$$
\mathbb{P}\left[H_{\partial \tilde{B}_{w_{0}}} \leq H_{\partial A_{2}}\right] \leq c \prod_{1 \leq i \leq d_{0}} \frac{w_{0}^{i}}{h} .
$$

This inequality can be proved using the independence of the random walk projections on the coordinate axes and rather standard techniques, and therefore we will omit its proof. It can be interpreted as a version of the gambler's ruin estimate (see Section 5.1 of Lawler and Limic, 2010) where each of the walk's projections has to reach distance $\sim h$ from $\partial A_{2}$ without ever hitting the associated projections of $\partial A_{2}$ in the process. 
We will change the notation so that we are able to express (A.20) in a way that does not uses the fact that $\{0\}^{d}$ is the vertex of $\{0, r+2 s\}^{d}$ which is closest to $w_{0}$. Recall the definition of $\mathfrak{H}_{i}^{d-1}, i=1, \ldots, 2 d$; the $(d-1)$-dimensional hyperfaces of $\mathfrak{H}_{r+2 s}$, of $l_{i}^{w_{0}}:=\min \left\{\operatorname{dist}\left(w_{0}, \mathfrak{H}_{i}^{d-1}\right), h\right\}$, and of $l_{i}^{y_{0}}:=\min \left\{\operatorname{dist}\left(y_{0}, \mathfrak{H}_{i}^{d-1}\right), h\right\}$. We have that (A.20) implies

$$
\mathbb{P}_{w_{0}}\left[H_{\partial \tilde{B}_{w_{0}}} \leq H_{\partial A_{2}}\right] \leq c \frac{l_{1}^{w_{0}} \ldots l_{2 d}^{w_{0}}}{h^{2 d}}
$$

Together with (A.19), this shows

$$
\mathbb{P}_{w_{0}}\left[w_{0} \stackrel{2^{\prime}}{\rightarrow} y_{0}\right] \leq h^{-(d-2)} \frac{l_{1}^{w_{0}} \ldots l_{2 d}^{w_{0}}}{h^{2 d}} \frac{l_{1}^{y_{0}} \ldots l_{2 d}^{y_{0}}}{h^{2 d}} .
$$

We need a matching lower bound. Again we assume $h>100 s$, since otherwise the lower bound follows immediately from using (2.1). We define

$$
w_{5}:=\left(w_{0}^{1}+\frac{h}{4 \sqrt{d}}, \ldots, w_{0}^{d_{0}}+\frac{h}{4 \sqrt{d}}, w_{0}^{d_{0}+1}, \ldots, w_{0}^{d}\right),
$$

We analogously define $y_{5}$ : Let $e_{i_{d_{1}}}, \ldots, e_{i_{d_{k}}}$ be the vectors in the orthonormal basis of $\mathbb{R}^{d}$ corresponding to the directions in which the ball $B_{\infty}\left(y_{0}, \frac{h}{4 \sqrt{d}}\right)$ passes the limits of the hypercube $\mathfrak{H}_{r+2 s} \cdot y_{5}$ is defined to be the point in $A_{2}^{C}$ such that

$$
\begin{gathered}
l=d_{1}, \ldots, d_{k} \Longrightarrow\left|\left\langle y_{5}-y_{0}, e_{i_{l}}\right\rangle\right|=\frac{h}{4 \sqrt{d}}, \\
n \neq d_{1}, \ldots, d_{k} \Longrightarrow\left|\left\langle y_{5}-y_{0}, e_{i_{n}}\right\rangle\right|=0 \\
\text { and } \\
B_{\infty}\left(y_{5}, \frac{h}{4 \sqrt{d}}\right) \subseteq \mathfrak{H}_{r+2 s} .
\end{gathered}
$$

Our plan is to describe an event contained in $w_{0} \stackrel{2^{\prime}}{\rightarrow} y_{0}$ with probability matching that of the right side of $(A .21)$. We let

$$
\hat{B}_{w_{5}}:=\hat{B}_{\infty}\left(w_{5}, \frac{h}{16 \sqrt{d}}\right)
$$

and

$$
\hat{B}_{y_{5}}:=\hat{B}_{\infty}\left(y_{5}, \frac{h}{16 \sqrt{d}}\right),
$$

For $w_{6} \in \partial \hat{B}_{w_{5}}$ and $y_{6} \in \partial \hat{B}_{y_{5}}$, we define the events

- $w_{0} \stackrel{8}{\rightarrow} w_{6}$ : The event where the random walk started at $w_{0}$ hits $\partial \hat{B}_{w_{5}}$ before hitting $\partial A_{2}$ and its entrance point in $\partial \hat{B}_{w_{5}}$ is $w_{6}$.

- $w_{6} \stackrel{9}{\rightarrow} y_{6}$ : The event where the random walk started at $w_{6}$ visits $y_{6} \in \partial \hat{B}_{y_{5}}$ before reaching $\partial A_{2}$.

- $y_{6} \stackrel{10}{\longrightarrow} y_{0}$ : The event where the simple random walk started at $y_{6}$ hits $y_{0}$ before returning to $\partial \hat{B}_{y_{5}}$. 


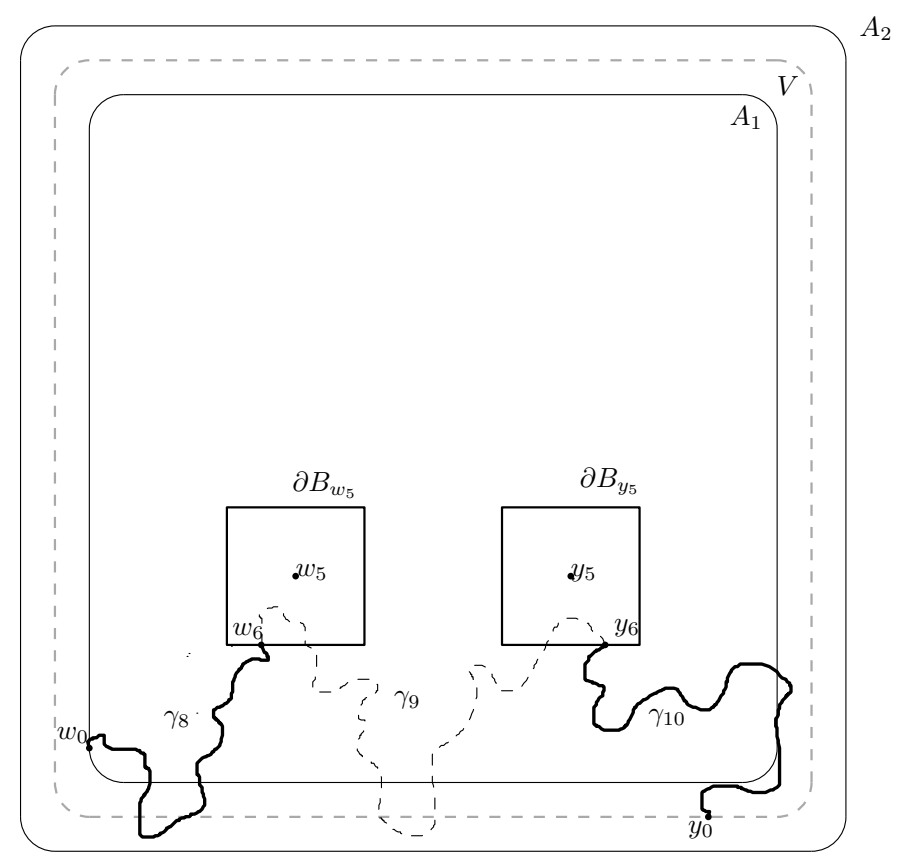

Figure A.10. Definition of the paths $\gamma_{8}, \gamma_{9}$ and $\gamma_{10}$.

And we denote by $w_{0} \stackrel{8}{\rightarrow} w_{6} \stackrel{9}{\rightarrow} y_{6} \stackrel{10}{\rightarrow} y_{0}$ the "concatenation" of these three events, that is, the path $\gamma$ belongs to the event $w_{0} \stackrel{8}{\rightarrow} w_{6} \stackrel{9}{\rightarrow} y_{6} \stackrel{10}{\rightarrow} y_{0}$ if and only if $\gamma$ is the concatenation of three paths: $\gamma_{8} \in w_{0} \stackrel{8}{\rightarrow} w_{6}, \gamma_{9} \in w_{6} \stackrel{9}{\rightarrow} y_{6}$ and $\gamma_{10} \in y_{6} \stackrel{10}{\longrightarrow} y_{0}$. It is then clear that

$$
\bigcup_{w_{6}} \bigcup_{y_{6}} w_{0} \stackrel{8}{\rightarrow} w_{6} \stackrel{9}{\rightarrow} y_{6} \stackrel{10}{\rightarrow} y_{0} \subset w_{0} \stackrel{2^{\prime}}{\rightarrow} y_{0},
$$

so that; summing over $\gamma_{8} \in w_{0} \stackrel{8}{\rightarrow} w_{6}, \gamma_{9} \in w_{6} \stackrel{9}{\rightarrow} y_{6}$ and $\gamma_{10} \in y_{6} \stackrel{10}{\rightarrow} y_{0}$; we have

$$
\begin{aligned}
\mathbb{P}_{w_{0}}\left[w_{0} \stackrel{2^{\prime}}{\rightarrow} y_{0}\right] & \geq \sum_{w_{6}} \sum_{\gamma_{8}} \frac{1}{(2 d)^{\left|\gamma_{8}\right|}} \sum_{y_{6}} \sum_{\gamma_{9}} \frac{1}{(2 d)^{\left|\gamma_{9}\right|}} \sum_{\gamma_{10}} \frac{1}{(2 d)^{\left|\gamma_{10}\right|}} \\
& =\sum_{w_{6}} \mathbb{P}_{w_{0}}\left[w_{0} \stackrel{8}{\rightarrow} w_{6}\right] \sum_{y_{6}} \mathbb{P}_{w_{6}}\left[w_{6} \stackrel{9}{\rightarrow} y_{6}\right] \mathbb{P}_{y_{6}}\left[y_{6} \stackrel{10}{\rightarrow} y_{0}\right] \\
& \geq \frac{c}{h^{d-2}} \sum_{w_{6}} \mathbb{P}_{w_{0}}\left[w_{0} \stackrel{8}{\rightarrow} w_{6}\right] \sum_{y_{6}} \mathbb{P}_{y_{6}}\left[y_{6} \stackrel{10}{\rightarrow} y_{0}\right]
\end{aligned}
$$

where we bounded $\mathbb{P}_{w_{6}}\left[w_{6} \stackrel{9}{\rightarrow} y_{6}\right]$ from below by $c h^{d-2}$ using a Green's function estimate and the fact that the distance of both $w_{6}$ and $y_{6}$ from $\partial A_{2}$ has order $h$.

Due to the simple random walk's reversibility, we obtain

$$
\mathbb{P}_{w_{0}}\left[w_{0} \stackrel{2^{\prime}}{\rightarrow} y_{0}\right] \geq \frac{c}{h^{d-2}} \mathbb{P}_{w_{0}}\left[H_{\partial \hat{B}_{w_{5}}}<H_{\partial A_{2}}\right] \mathbb{P}_{y_{0}}\left[H_{\partial \hat{B}_{y_{5}}}<H_{\partial A_{2}}\right]
$$


The bound for $\mathbb{P}_{w_{0}}\left[H_{\partial \hat{B}_{w_{5}}}<H_{\partial A_{2}}\right]$ and the bound for $\mathbb{P}_{y_{0}}\left[H_{\partial \hat{B}_{y_{5}}}<H_{\partial A_{2}}\right]$ are obtained in the same way. All we need is the following inequality:

$$
\mathbb{P}_{w_{0}}\left[H_{\partial \hat{B}_{w_{5}}}<H_{\partial A_{2}}\right] \geq c \prod_{1 \leq i \leq d_{0}} \frac{w_{0}^{i}}{h}
$$

but again this bound follows from standard arguments from random walk theory, where we essentially use the independence of the random walk projections, the gambler's ruin estimate (Section 5.1 of Lawler and Limic, 2010) and the central limit theorem in order to construct an event where all random walk projections hit the respective projections of $\partial \hat{B}_{w_{5}}$ at around the same time without ever hitting the projections of $\partial A_{2}$. We will therefore omit the details.

Together with (A.24), (A.25) implies, using the general notation,

$c h^{-(d-2)} \frac{l_{1}^{w_{0}} \ldots l_{2 d}^{w_{0}}}{h^{2 d}} \cdot \frac{l_{1}^{y_{0}} \ldots l_{2 d}^{y_{0}}}{h^{2 d}} \leq \mathbb{P}_{w_{0}}\left[w_{0} \stackrel{2^{\prime}}{\rightarrow} y_{0}\right] \leq c^{\prime} h^{-(d-2)} \frac{l_{1}^{w_{0}} \ldots l_{2 d}^{w_{0}}}{h^{2 d}} \cdot \frac{l_{1}^{y_{0}} \ldots l_{2 d}^{y_{0}}}{h^{2 d}}$.

$\boldsymbol{w} \stackrel{4}{\rightarrow} \boldsymbol{y}:$ We suppose $h_{4}>100 s$, since $(2.1)$ implies the case when $h_{4}<100 s$. We will start with the lower bound. Let $B_{1}$ be a discrete Euclidean ball of radius $s$ contained in $A_{2}^{C}$ such that $\partial A_{2} \cap B_{1}=\{y\}$. Let $B_{2}$ be a discrete Euclidean ball of radius $\frac{s}{4}$ concentric with $B_{1}$. Then, the probability that the walk started at $y$ hits $B_{2}$ before returning to $\partial A_{2}$ is bigger than the probability that it hits $B_{2}$ before returning to $B_{1}$, and has order $s^{-1}$. Now, for every point $\tilde{y} \in \partial B_{2}$, we bound $\mathbb{P}_{w}\left[w \stackrel{2^{\prime}}{\rightarrow} \tilde{y}\right]$ from below in exactly the same way as we bounded $\mathbb{P}_{w_{0}}\left[w_{0} \stackrel{2^{\prime}}{\rightarrow} y_{0}\right]$. So that, using the walk's reversibility, the fact that $h_{4}>100 s$, and the same notation introduced above, we have

$$
\begin{aligned}
\mathbb{P}_{w}[w \stackrel{4}{\rightarrow} y] & \geq \sum_{\tilde{y} \in \partial B_{2}} \mathbb{P}_{w}\left[w \stackrel{2^{\prime}}{\rightarrow} \tilde{y}\right] \mathbb{P}_{y}\left[H_{\partial B_{2}}<H_{\partial A_{2}}, X_{H_{\partial B_{2}}}=\tilde{y}\right] \\
& \geq c s^{-1} \inf _{\tilde{y} \in \partial B_{2}} \mathbb{P}_{w}\left[w \stackrel{2^{\prime}}{\rightarrow} \tilde{y}\right] \\
& \geq c s^{-1} h_{4}^{-(d-2)} \inf _{\tilde{y} \in \partial B_{2}} \frac{l_{1}^{w_{0}} \ldots l_{2 d}^{w_{0}}}{h_{4}^{2 d}} \cdot \frac{l_{1}^{\tilde{y}} \ldots l_{2 d}^{\tilde{y}}}{h_{4}^{2 d}} .
\end{aligned}
$$

For the upper bound, let $B_{1}^{\prime}$ be a discrete Euclidean ball of radius $s$ contained in $A_{2} \cup \partial A_{2}$ such that $\partial A_{2} \cap B_{1}^{\prime}=\{y\}$. Let $B_{2}^{\prime}$ be a discrete Euclidean ball of radius $2 s$ concentric with $B_{1}^{\prime}$. Then

$$
\begin{aligned}
\mathbb{P}_{w}[w \stackrel{4}{\rightarrow} y] & \leq \sum_{\hat{y} \in \partial B_{2}^{\prime}} \mathbb{P}_{w}\left[w \stackrel{2^{\prime}}{\rightarrow} \hat{y}\right] \mathbb{P}_{y}\left[H_{\partial B_{2}^{\prime}}<H_{\partial A_{2}}, X_{H_{\partial B_{2}^{\prime}}}=\hat{y}\right] \\
& \leq c s^{-1} \inf _{\hat{y} \in \partial B_{2}^{\prime}} \mathbb{P}_{w}\left[w \stackrel{2^{\prime}}{\rightarrow} \hat{y}\right] \\
& \leq c s^{-1} h_{4}^{-(d-2)} \sup _{\hat{y} \in \partial B_{2}^{\prime}} \frac{l_{1}^{w_{0}} \ldots l_{2 d}^{w_{0}}}{h_{4}^{2 d}} \frac{l_{1}^{\hat{y}} \ldots l_{2 d}^{\hat{y}}}{h_{4}^{2 d}} .
\end{aligned}
$$

We have proved the following proposition:

Proposition A.2. Regarding the sets $A_{1}^{\square}, V^{\square}$ and $A_{2}^{\square}$, we have that, using the notation defined above, for some constants $c_{1}, c_{2}, c_{3}, c_{4}, c_{5}, c_{6}, c_{7}, c_{8}, c_{9}>0$, the 
following bounds are valid:

$$
\begin{gathered}
c_{1} e^{\frac{-c_{2} h_{1}}{s}} s^{-(d-1)} \leq \mathbb{P}_{w}\left[w \stackrel{1}{\rightarrow} w_{0}\right] \leq c_{2} e^{\frac{-c_{2} h_{1}}{s}} s^{-(d-1)}, \\
c_{3} e^{\frac{-c_{4} h_{3}}{s}} s^{-(d-1)} s^{-1} \leq \mathbb{P}_{w}\left[y \stackrel{3}{\rightarrow} y_{0}\right] \leq c_{4} e^{\frac{-c_{4} h_{3}}{s}} s^{-(d-1)} s^{-1} \\
c_{5} h^{-(d-2)} \frac{l_{1}^{w_{0}} \ldots l_{2 d}^{w_{0}}}{h^{2 d}} \frac{l_{1}^{y_{0}} \ldots l_{2 d}^{y_{0}}}{h^{2 d}} \leq \mathbb{P}_{w_{0}}\left[w_{0} \stackrel{2^{\prime}}{\rightarrow} y_{0}\right] \leq c_{6} h^{-(d-2)} \frac{l_{1}^{w_{0}} \ldots l_{2 d}^{w_{0}}}{h^{2 d}} \frac{l_{1}^{y_{0}} \ldots l_{2 d}^{y_{0}}}{h^{2 d}}, \\
c_{7} s^{-1} h_{4}^{-(d-2)} \inf _{\tilde{y} \in \partial B_{2}} \frac{l_{1}^{w_{0}} \ldots l_{2 d}^{w_{0}}}{h_{4}^{2 d}} \frac{l_{1}^{\tilde{y}} \ldots l_{2 d}^{\tilde{y}}}{h_{4}^{2 d}} \leq \mathbb{P}_{w_{0}}[w \stackrel{4}{\rightarrow} y] \\
\leq c_{8} s^{-1} h_{4}^{-(d-2)} \sup _{\hat{y} \in \partial B_{2}^{\prime}} \frac{l_{1}^{w_{0}} \ldots l_{2 d}^{w_{0}}}{h_{4}^{2 d}} \frac{l_{1}^{\hat{y}} \ldots l_{2 d}^{\hat{y}}}{h_{4}^{2 d}} . \\
\sup _{\substack{w \in V \\
y \in \partial A_{2}}} \mathbb{P}_{w}\left[w \stackrel{1}{\rightarrow} w_{0} \stackrel{2}{\rightarrow} y_{0} \stackrel{3}{\rightarrow} y \mid w \stackrel{4}{\rightarrow} y\right] \leq c_{9} s^{-2(d-1)} .
\end{gathered}
$$

A.2. Proof of Lemma 4.5. Let $z \in \Sigma$ be such that $\Xi(z)=\left(w_{0}, y_{0}\right)$, and again let $h$ stand for the Euclidean distance between $w_{0}$ and $y_{0}$. We let $\pi\left(w_{0}, y_{0}\right)$ be defined in the same way as in (4.5). Given a simple random walk trajectory $\varrho$ started in a set $B$ containing $V$, we define $\mathcal{C}_{w_{0}, y_{0}}^{B}(\varrho)$ to be the function that counts how many times the random walk trajectory $\varrho$ makes an excursion on $A_{2}^{C}$ that enters $A_{1}$ at $w_{0}$, and $y_{0}$ is the last point such excursion visits on $V$ before reaching $\partial A_{2}$. We let $\mathcal{C}_{w_{0}, y_{0}}^{B}$ denote the random variable $\mathcal{C}_{w_{0}, y_{0}}^{B}(\bar{\varrho})$ when $\varrho$ 's first point is chosen according to $\bar{e}_{B}$. Proposition 3.3 then implies

$$
\pi\left(w_{0}, y_{0}\right)=\mathbb{E}\left(\mathcal{C}_{w_{0}, y_{0}}^{V}\right)
$$

Define $\tilde{V}:=\partial B(0,3(r+s))$, the discrete sphere of radius $3(r+s)$. We define

$$
\tilde{\pi}\left(w_{0}, y_{0}\right):=\mathbb{E}\left(\mathcal{C}_{w_{0}, y_{0}}^{\tilde{V}}\right) .
$$

From the compatibility of the laws defined in (2.3), one can see that (see also the proof of Lemma 6.2 of Popov and Teixeira, 2015):

$$
u \operatorname{cap}(\tilde{V}) \mathbb{E}\left(\mathcal{C}_{w_{0}, y_{0}}^{\tilde{V}}\right)=u \operatorname{cap}(V) \mathbb{E}\left(\mathcal{C}_{w_{0}, y_{0}}^{V}\right) .
$$

Since $\operatorname{cap}(\tilde{V}) \asymp \operatorname{cap}(V)$, if we successfully estimate $\tilde{\pi}\left(w_{0}, y_{0}\right)$ we will automatically be provided with an estimate for $\pi\left(w_{0}, y_{0}\right)$. We changed the problem from estimating $\pi\left(w_{0}, y_{0}\right)$ to estimating $\tilde{\pi}\left(w_{0}, y_{0}\right)$ so that the distance between the simple random walk's starting point and $w_{0}$ does not affect our calculations.

First we note that $\mathcal{C}_{w_{0}, y_{0}}^{\tilde{V}}$ is dominated by a Geometric $\left(c_{1}\right)$ random variable, for some $0<c_{1}<1$. This follows from the fact that every time the simple random walk exits $A_{2}^{C}$, with probability uniformly greater than some constant $1-c_{1}>0$, the walk never returns to $w_{0}$. This way, it will be sufficient to estimate the probability $\mathcal{P}\left[\mathcal{C}_{w_{0}, y_{0}}^{\tilde{V}} \geq 1\right]$ for our purposes.

So, for a walk started at $\tilde{V}$ to reach $w_{0}$, it first has to hit a discrete sphere $\partial B_{1}$ of radius $\frac{s}{2}$ centered on $w_{0}$. The probability of such event is of order $\frac{s^{d-2}}{r^{d-2}}$, by Proposition 6.4.2 of Lawler and Limic (2010).

Let $B_{2}$ be a discrete Euclidean ball of radius $s$ contained in $A_{1}$ such that $B_{2} \cap$ $A_{1}=\left\{w_{0}\right\}$. We also let $B_{3}$ be a discrete Euclidean ball of radius $2 s$ lying outside 
$A_{1}$ such that $B_{3} \cap A_{1}=\left\{w_{0}\right\}$. Using Proposition 6.5.4 of Lawler and Limic (2010) we have, for any $x^{\prime} \in \partial B_{1} \cap A_{!}^{C}$ and some constant $c_{2}>0$ :

$$
\mathbb{P}_{x^{\prime}}\left[X_{H_{A_{1}}}=w_{0}\right] \leq \mathbb{P}_{x^{\prime}}\left[X_{H_{B_{2}}}=w_{0}\right] \leq c_{2} s^{-(d-1)} .
$$

Then, recalling the notation $f_{A_{1}}\left(w_{0}, y_{0}\right):=\mathbb{P}_{w_{0}}\left[w_{0} \stackrel{2^{\prime}}{\rightarrow} y_{0}\right]$ and the fact that $\operatorname{cap}(V) \asymp r^{(d-2)}$, and using the strong Markov property, we get, for constants $c, c_{1}>0$ :

$$
\pi\left(w_{0}, y_{0}\right) \leq c \mathcal{P}\left[\mathcal{C}_{w_{0}, y_{0}}^{\tilde{V}} \geq 1\right] \leq c_{1} \operatorname{cap}(V)^{-1} s^{-1} f_{A_{1}}\left(w_{0}, y_{0}\right) .
$$

For the lower bound, we let $B_{4}$ be a discrete Euclidean ball of radius $\frac{s}{4}$ contained in $A_{2}^{C} \backslash B(0, r+s)$ such that for every $x \in B_{4}$, $\operatorname{dist}\left(x, w_{0}\right) \leq 2 s$. Using the strong Markov property, we get

$$
\mathcal{P}\left[\mathcal{C}_{w_{0}, y_{0}}^{\tilde{V}} \geq 1\right] \geq \inf _{x \in \tilde{V}} \mathbb{P}_{x}\left[H_{B_{4}}<\infty\right] \inf _{x^{\prime \prime} \in B_{4}} \mathbb{P}_{x}\left[X_{B_{3}}=w_{0}\right] f_{A_{1}}\left(w_{0}, y_{0}\right),
$$

so that, using Proposition 6.4.2 of Lawler and Limic (2010) we have, for some constant $c_{3}>0$,

$$
\pi\left(w_{0}, y_{0}\right) \geq c_{3} \operatorname{cap}(V)^{-1} s^{-1} f_{A_{1}}\left(w_{0}, y_{0}\right) .
$$

The part (ii) then follows from (i) and Proposition 3.4.

A.3. A lower bound for $\alpha$. Let $z \in \Sigma$ be such that $\Xi(z)=\left(w_{0}, y_{0}\right)$, let $c_{4}>0$ be some positive real number. For

$$
\Gamma_{w_{0}, y_{0}}:=\left\{\left(w_{0}^{\prime}, y_{0}^{\prime}\right) \in V \times \partial A_{2} ; \max \left\{\left\|w_{0}^{\prime}-w_{0}\right\|,\left\|y_{0}^{\prime}-y_{0}\right\|\right\} \leq c_{4} s\right\}
$$

and

$$
\alpha:=\inf \left\{\frac{g_{(w, y)}\left(z^{\prime}\right)}{g_{(w, y)}(\hat{z})} ;(w, y) \in V \times \partial A_{2}, z^{\prime} \in \Gamma_{w_{0}, y_{0}}, \hat{z} \in \mathcal{K}\right\},
$$

we need to find a constant lower bound for $\alpha$. But this is just a consequence of the bounds obtained in Propositions A.1 and A.2.

\section{Acknowledgments}

Caio Alves was supported by FAPESP (grants 2013/24928-2 and 2015/18930-0). Serguei Popov was supported by CNPq (grant 300886/2008) and FAPESP (grant 2009/523798).

\section{References}

N. Berger, N. Gantert and Y. Peres. The speed of biased random walk on percolation clusters. Probab. Theory Related Fields 126 (2), 221-242 (2003). MR1990055.

D. de Bernardini, C. Gallesco and S. Popov. An improved decoupling inequality for random interlacements $(2018+)$. Work in progress.

J. Černý and A. Teixeira. From random walk trajectories to random interlacements, volume 23 of Ensaios Matemáticos [Mathematical Surveys]. Sociedade Brasileira de Matemática, Rio de Janeiro (2012). ISBN 978-85-85818-69-2. MR3014964.

J. Černý and A. Teixeira. Random walks on torus and random interlacements: macroscopic coupling and phase transition. Ann. Appl. Probab. 26 (5), 28832914 (2016). MR3563197. 
Y. Chang and A. Sapozhnikov. Phase transition in loop percolation. Probab. Theory Related Fields 164 (3-4), 979-1025 (2016). MR3477785.

A. Drewitz, B. Ráth and A. Sapozhnikov. An introduction to random interlacements. SpringerBriefs in Mathematics. Springer, Cham (2014). ISBN 978-3-31905851-1; 978-3-319-05852-8. MR3308116.

A. Fribergh. Biased random walk in positive random conductances on $\mathbb{Z}^{d}$. Ann. Probab. 41 (6), 3910-3972 (2013). MR3161466.

A. Fribergh and A. Hammond. Phase transition for the speed of the biased random walk on the supercritical percolation cluster. Comm. Pure Appl. Math. 67 (2), 173-245 (2014). MR3149843.

A. Fribergh and S. Popov. Biased random walks on the interlacement set. Ann. Inst. Henri Poincaré Probab. Stat. 54 (3), 1341-1358 (2018). MR3825884.

G. F. Lawler. Intersections of random walks. Modern Birkhäuser Classics. Birkhäuser/Springer, New York (2013). ISBN 978-1-4614-5971-2; 978-1-46145972-9. MR2985195.

G. F. Lawler and V. Limic. Random walk: a modern introduction, volume 123 of Cambridge Studies in Advanced Mathematics. Cambridge University Press, Cambridge (2010). ISBN 978-0-521-51918-2. MR2677157.

S. Popov and B. Ráth. On decoupling inequalities and percolation of excursion sets of the Gaussian free field. J. Stat. Phys. 159 (2), 312-320 (2015). MR3325312.

S. Popov and A. Teixeira. Soft local times and decoupling of random interlacements. J. Eur. Math. Soc. (JEMS) 17 (10), 2545-2593 (2015). MR3420516.

E. B. Procaccia, R. Rosenthal and A. Sapozhnikov. Quenched invariance principle for simple random walk on clusters in correlated percolation models. Probab. Theory Related Fields 166 (3-4), 619-657 (2016). MR3568036.

S. I. Resnick. Extreme values, regular variation and point processes. Springer Series in Operations Research and Financial Engineering. Springer, New York (2008). ISBN 978-0-387-75952-4. MR2364939.

A.-S. Sznitman. On the anisotropic walk on the supercritical percolation cluster. Comm. Math. Phys. 240 (1-2), 123-148 (2003). MR2004982.

A.-S. Sznitman. Vacant set of random interlacements and percolation. Ann. of Math. (2) 171 (3), 2039-2087 (2010). MR2680403.

A.-S. Sznitman. Decoupling inequalities and interlacement percolation on $G \times \mathbb{Z}$. Invent. Math. 187 (3), 645-706 (2012a). MR2891880.

A.-S. Sznitman. An isomorphism theorem for random interlacements. Electron. Commun. Probab. 17, no. 9, 9 (2012b). MR2892408.

A.-S. Sznitman. Topics in occupation times and Gaussian free fields. Zurich Lectures in Advanced Mathematics. European Mathematical Society (EMS), Zürich (2012c). ISBN 978-3-03719-109-5. MR2932978.

A. Teixeira. Interlacement percolation on transient weighted graphs. Electron. J. Probab. 14, no. 54, 1604-1628 (2009). MR2525105. 